\title{
Design and Evaluation of a Real Time Physiological Signals Acquisition System Implemented in Multi-Operating Rooms for Anesthesia
}

\author{
Quan Liu ${ }^{1,2}$, Li Ma ${ }^{1,2}$, Shou-Zen Fan ${ }^{3}$, Maysam F. Abbod ${ }^{4}$, Cheng-Wei Lu,6, Tzu-Yu Lin ${ }^{5,6}$, Kuo- \\ Kuang $\mathrm{Jen}^{7}$, Shang-Ju $\mathrm{Wu}^{7}$, Jiann-Shing Shieh ${ }^{5, *}$ \\ 1 School of Information Engineering, Wuhan University of Technology, Wuhan 430070, China; \\ 2 Key Laboratory of Fiber Optic Sensing Technology and Information Processing, Ministry of Education, \\ Wuhan 430070, China \\ E-Mail: quanliu@whut.edu.cn (Q.L.); excellentmary@whut.edu.cn (L.M.) \\ 3 Department of Anesthesiology, College of Medicine, National Taiwan University, Taipei 100, Taiwan; E- \\ Mail: shouzen@gmail.com; \\ 4 Department of Electronic and Computer Engineering, Brunel University London, UB8 3PH, UK; \\ E-Mail: maysam.abbod@brunel.ac.uk; \\ 5 Department of Mechanical Engineering, and Innovation Center for Big Data and Digital Convergence, Yuan \\ Ze University, 135, Yuan-Tung Road, Chung-Li 32003, Taiwan; \\ 6 Department of Anesthesiology, Far Eastern Memorial Hospital, Ban-Chiao, Taiwan; \\ drluchengwei@gmail.com (C.-W. L.); drlin1971@gmail.com(T.-Y. L.); \\ 7 National Chung-Shan Institute of Science and Technology, Taoyuan, Longtan Taiwan; rgg@ms7.hinet.net \\ (K.-K. J.); shanglu111@gmail.com (S.-J. W.);
}

* Author to whom correspondence should be addressed; E-Mail: jsshieh@saturn.yzu.edu.tw; Tel.: +886-3-4638800 ext. 2470; Fax: +886-3-4558013.

\begin{abstract}
With critical importance of medical healthcare, there exist urgent needs for in-depth medical studies that can access and analyze specific physiological signals to provide theoretical support for practical clinical care. As a consequence, obtaining the valuable medical data with minimal cost and impacts on hospital work comes as the first concern of researchers. Anesthesia plays a widely recognized role in surgeries, which attracts people to undertake relevant research. In this paper, a real-time physiological medical signal data acquisition system (PMSDA) for the multioperating room applications is proposed with high universality of the hospital practical settings and research requirements. By utilizing a wireless communication approach, it provides an easily accessible network platform for collection of physiological medical signals such as photoplethysmogram (PPG), electrocardiograph (ECG) and electroencephalogram (EEG) during the surgery. In addition, the raw data is stored on a server for safe backup and further analysis of depth of anesthesia (DoA). Results show that the PMSDA exhibits robust, high quality performance and efficiently reduces costs, as compared to previously used manual methods and allow seamless integration into hospital environment, independent of its routine work. Overall, it provides a pragmatic and flexible surgery-data acquisition system model with minimum impact and resource cost applicable to research in critical and practical medical circumstances.
\end{abstract}

Keywords anesthesia, data acquiring, flexible, multi-operating room, physiological medical signal, wireless network platform. 


\section{Introduction}

Over the past few decades, demand for medical service of high quality has rapidly increased. To cope with this scenario, large amounts of physiological data is proposed to be captured and analyzed in order to provide the scientific support for intelligent care [1,2]. Advances in modern computer system and medical sensor technology support this goal, potentially offering an unprecedented view of physiologic subtlety and variability in health and disease [3]. In particular, the world has stepped into the data-dependent age, which is often termed as Big Data [4], e.g. Hadoop, Spark, and Storm, the most commonly used distributed Big Data platforms [5-7]. As a result, many hospitals upgraded their Hospital Information System (HIS) in the past decades, which integrates the Electronic Health Records (EHRs), to store a variety of data resource such as life physiological data, clinical data, administrative data and social network data for better medical care quality and management [8-10].

Although the HIS accumulates tremendous amount data, it does not perform well in application to specific disease modeling, prediction and treatment $[4,11]$. In other words, its main focus remains to be on routine hospital work, rather than medical research. Additionally, most of HIS, including advanced systems, only store routine intermittent observations, not continuous waveforms of high fidelity due to storage costs. Finally, researchers are not likely to access this data because of concerns of system security and stability in surgery, patients' privacy, and access auxiliary tool fees charged by the system provider, interface communication software purchase. These concerns may explain why most clinical research groups still employ standalone equipment for data collection.

In order to overcome these drawbacks and inconvenience for some applications to specific diseases, several works have been conducted. PhysioNet [12] developed by Harvard University provides a large collection of recorded physiological signals (PhysioBank) and related open-source software (PhysioToolkit) [13]. It focuses on the studies of cardiovascular time series dynamics. Another example is a multimodal neuro-intensive care monitoring system has been established and adopted by the Cambridge Neurosurgical Unit, UK [14]. In addition to the detection of multiple parameters of head injury cases, it also presents the dynamics of the studied pathology and proposes useful secondary indexes for clinical neuroscience research. Both databases focus on specific diagnosis applications: cardiovascular dynamics and head injuries instead of details of the collection methods based on their published works. Such methods likely require large amounts of funds and resources. Thus, they are not viable by small groups or individuals, regardless of their specific medical field, e.g. anesthesia. This leaves a critical unfulfilled need for a flexible and low-cost solution for obtaining this data of interest.

Anesthesia is a kind of temporary drug-induced and reversible state associated with a loss of consciousness or sedation under doctor's manipulation by the controlling the doses of anesthetics [15]. Its effects include paralysis, meaning muscle relaxation; analgesia, representing pain relief and amnesia, symbolizing memory loss or unconsciousness. Undoubtedly, anesthesia is crucial to safety in most surgical and many nonsurgical procedures. Since the first public demonstration of anesthesia operation in Massachusetts General Hospital in 1846, it soon became fundamental and necessary component of surgeries, accomplishing elimination of the patients' pain by blocking his/her nerve receptors [16]. To this day, the underlying mechanisms from cellar level to system level, as well as methods of evaluation of depth of anesthesia remain unclear [17-19]. Thus, it remains an active area of research. Still, to facilitate such studies of anesthesia mechanisms, a method and efficient infrastructure for collection of relevant high quality data is critically needed.

Since so many groups are dedicated to anesthesia research, the anesthesia physiological data format and structure may vary. An absence of standardized methodology for collecting data can affect the research issues related to anesthesia, lays a barrier to universal advances and replicability in this area [20]. So, increasing the standard reliability becomes extremely important in modern advanced care monitoring and anesthesia data collection systems. However, in commercial healthcaring surroundings, collecting more complete data is not the top priority of health providers in hospitals, resulting in the data heterogeneity by different research groups [21,22]. Hence, a reliable system that simplifies and automates the collecting process is necessary.

To address the issue described above, our research aims at obtaining clinical physiological data including electroencephalography (EEG), electrocardiography (ECG), photoplethysmogram (PPG), peripheral capillary oxygen saturation (SpO2), blood pressure (BP) and other signals to be stored for sharing, mining and analyzing, especially focusing on the depth of anesthesia (DoA) application. The data source is derived from the most widely applied non-invasive machine: IntelliVue MP60 (Philips, Germany), thus potentially providing assurance of data quality and standard structure 
format.

This paper is organized as follows: Section 2 roughly explains the related work about hospital environment and describes the system architecture, including the construction of the hardware platform as well as the software design. Section 3 shows the outcomes and performance of the system. Section 4 illustrates discussion about the limitations and future works. In Section 5, the conclusion is summarized.

\section{Materials and methods}

\subsection{Materials Patients and Previous Works}

This study protocol is approved by the participated hospitals: National Taiwan University Hospital (NTUH) and National Taiwan University Children Hospital (NTUCH) both located in Taipei City, Taiwan. The data was recorded from routine clinical multimodality monitoring via the MP60 as seen in Fig. 1. The informed consents under anesthesia are obtained from all patients. Fig. 2 illustrates the general layout of the operation rooms. In the practical operation environment, space occupied by medical equipment, working staff and some accessories appears much more crowded. To guarantee the patients' operation safety, our design should minimize the impact on surgery work. Stability and security become the top priority of the whole design procedure.

This paper's work intends to improve the efficiency of acquiring anesthesia physiological data because the data collection was conducted manually previously. Fig. 3 shows the structure of original collection framework [23]. The procedure is quite complex and time consuming. A laptop should be prepared, which is connected by the medical monitor via serial port cable after placement of all sensors. Once finishing the collection, we will manually categorize the data and duplicate them to the hard disk back in laboratory. Besides, the permission for entering operation room should be applied in advance. Only three to five cases can be obtained per day under the standard conditions, leading to an extremely low collection rate. Each time for data collection, it costs a lot of human energy, time, funds and so forth. As a result, this poses a requirement for system's optimization improvements.

\subsection{System Architecture}

In order to establish the infrastructure for anesthesia physiological signal and guarantee the data acquisition efficiency and effectiveness, we basically employ the mature and standard technique to keep the system stability instead of the aspect of advancement under the consideration of practical surgery environment.

Fig. 4 gives the overview of the new system working flow design. Rooms are connected to the data collection transfer station in control room through the local wireless network. Here, we take one room for example. To begin with, after the interaction with monitors, data obtained from the surface sensors will be labeled and put into data packet by the collection transfer station, followed by transferring the data to the specified database for backup storage, downloading, research sharing, etc. In adopting this method, the human's interference should diminish as much as possible. The detailed work will be separated into sections for explanation, together with the outcome in the result part.

\subsubsection{Infrastructure Platform}

In the light of similar surrounding situations in the two hospitals, a system is commissioned in NTUH as shown in Fig. 5. The physiological medical signal data acquisition system (PMSDA) system hardware platform is composed of surface sensors, front-end device, network complex, data transfer station and server. After the data is recorded by front-end device via kinds of sensors, physiological data is wirelessly transmitted through proxy transmitting module by access point (AP) and local gateway. The Control Room, one administrative room in hospital, serves as the data transfer station. Computers are provided there for pre-processing and uploading to the database server by our software installed inside it. Specifications of the components are as follows:

\section{Network Adoption}

Among all the components, the network framework is the top concern. In comparison with the manual method, the new design adopts the network to access mutli-operation rooms' data. Owing to the critical safety requirements by both hospital regulations, it is undoubtedly that appropriate option of network type counts very much. We surveyed the characteristics of different types of 
mainstream wireless networks. Wide area networks behave their own properties independent of healthcare applications. In Table 1, the characteristics of the most popular wireless connectivity technologies and protocols proposed in medical monitoring systems are listed [24].

- Low power consumption and small device size, short-range communication methods like Bluetooth and ZigBee are typically used with sensors in medical data collection. However, it presents capability of low data rate and short transmission distance, which is similar to Near Field Communication (NFC) as shown in Table 1. Besides, Zigbee platforms suffer from strong interference by WLANs, which share the same spectrum and transmit with larger signal power [25].

- WLAN technologies are avoided as low power sensor nodes because of their large size and power consumption. By contrast, it can provide longer ranges, i.e. $100 \mathrm{~m}$. And there are many commercial WiFi for patient and hospital staff to connect smart phone, tablet, etc. It proves the deployment convenience and compatible connection. In addition, its high speed guarantees any potential updates and optimization improvements.

- The short-range, low-data rate, UltraWideband (UWB) technology is another attractive technology that could be applied because of its regulated low transmitter power [26]. However, it has similar situation like the previous two types: Zigbee \& Bluetooth.

- In addition to unlicensed industrial, scientific and medical (ISM) bands, there are medical bands Wireless Medical Telemetry Service (WMTS) that are specifically regulated for medical monitoring by communication commissions around the world. But the data rate is not high enough, even lower than Zigbee. Furthermore, the frequency is not compatible for direct connection as well.

Through the efforts to review the current attempts in wireless technology, the PMSDA adopts the wireless local area network (WLAN) to build the wireless part of the infrastructure. Although interference might be encountered, WLAN has advantages of long transmission distance, high speed rate, flexible to connect, easy to detect and access. As to the matter of coexistence with other WLAN networks, the auto channel section mode can be activated. Also, we designed our own unique packet transmission protocol. As a whole, the convenience of WLAN can promote the deployment of the proposed system.

\section{Body Sensors}

We use kinds of sensors compatible with monitor MP60 for signal recording. PPG signal comes from M1191A (Philips, US) based on the changes measurement in red and infrared light absorption by tissue. It is attached to 8 pin connector with sampling rate $128 \mathrm{~Hz}$. ECG traces are sampled with $512 \mathrm{~Hz}$ by 5-leads cable sensor module M1621A (Philips, Germany). Note that this block provides respiration data simultaneously with $64 \mathrm{~Hz}$ sample frequency. By contrast, one channel EEG series is captured by 4 Quatro electrodes connected with Bispectral Index (BIS) module M1034 (Philips, Germany) through Power Link. We acquire the EEG data by appropriate location placement of sensors along with glue to reduce the impedance. The EEG sample rate is $128 \mathrm{~Hz}$. The cuff M1574A (Philips, Germany) is used to measure the noninvasive blood pressure. Besides, all the discrete vital sign indexes are derived from the corresponding waveform every 5 seconds such as Heart Rate from ECG, Pulse Rate from PPG, etc., which are consistent with BIS value sampling rate.

\section{Vital Signs Monitor}

As explained above, PMSDA system is an update extension of the previous one. In other words, our focus is not the specific device design, but the standard convenient and feasible clinical data collection model and DoA analysis in future. The veracity and standard should be widely recognized. Considering the multi-room connectivity and easy implementation of our system, it is reasonably to utilize the existing mainstream machines in every operating room, which are proved to be accurate by clinical doctors. Philips IntelliVue MP60 (Fig. 1) is treated as one of the most popular standard clinical medical devices for anesthesia monitoring, and can stably detect ECG, EEG, SPO2, PPG, $\mathrm{BP}$, etc. It supports the standard serial communication port to export the raw data, which make it sense that we can acquire the clinical physiological data for research. Therefore, MP60 makes the ideal choice together with compatible sensors explained above.

\section{Wireless Transmission Complex}

Fig. 6 shows the NPort W2250A, which is produced by MOXA Company. It serves for connecting serial or Ethernet to IEEE 802.11a/b/g network with secure methods of WEP, WPA, WPA2 [27]. In PMSDA system, NPort W2250A is connected to vital signs monitor machine data export port via serial cable with 115200 baud rate, no parity, 1 stop bit, and no software/hardware flow control. 
Then the exported data is transmitted to AP/repeater named D-Link N300 (D-Link, Taiwan) in Fig. 5 with highest wireless speed of $300 \mathrm{Mbps}$ and a $5 \mathrm{dBi}$ gain antenna. All the parameters and attributes can be set to meet the practical requirements such as range, channel and secure method by configuring web-page built-in. This stage performs a transparent transmission between serial and Intranet port. Any valid data needs the request and permission by the control software, i.e., the data collection software in application layer, which is developed in data collection transfer station by us.

\section{Terminal Equipment}

Computer and server are essential for this system. In the hospital control room, the computer works as data collection and pre-processing center to gather all nodes of the network. Then it resends the data to the server. The server is located in NTUH for this proprietary purpose, which can be visited through the Internet. General hardware performance could fulfill the demands, while the core is the software advancement.

\subsubsection{Software Organization}

The general layer block architecture is presented in Fig. 7, which has four layers performing different functions. The first and second layers need software configuration. The first layer is used to set vital signs monitor parameters in relation to communication like baud rate, output port selection, and signal category, etc. The second layer is the configuration of MOXA NPort W2250A device to convert data transmitting protocol from the serial port into wireless mode. This part is critical for the handshake with vital signs monitor and the link recognition to AP. Strictly speaking, PMSDA system programming software consists of the third and fourth layers. The third layer is the collection and pre-processing software installed on computer in control room. It behaves functions of handshake, room allocation and ID recognition with MOXA device as a gathering terminal point. The fourth layer is the physiological database server to provide services of data management, authority permission and guest visit. All of the layers run smoothly to acquire the intact medical signal data flow seamlessly.

\section{Data Collection}

The software, developed by $\mathrm{C}++$, undertakes the interaction with monitor machine based on the flow chart in Fig. 8. After initialization, network ping should be sent to guarantee the loop circulation. And then handshake association procedure is accomplished based on the manufacturer order. Only when the handshake and recognition is verified successfully, the connection can be valid. Next, different kinds of data are exported by the machine based on requests protocol followed by the data parsing stage. By releasing the connection, the data transmission comes to an end.

Next, through hundreds of trials of de-compilation of the data flow from the monitor, we check, recognize and extract the vital signs and its waveform value, getting the packet format structure in Fig. 9. The frame consists of five sections. Similar to standard packet frame, the beginning of frame (BOF) and the end of frame (EOF) are set a permanent value respectively with 1 byte each for data segment integrity check. Head information (Hdr) contains 3 attributes: 1 byte protocol id containing ID and version information; 1 byte message type, indicating the message class like association control, lifetick, message conformation, etc; 2 bytes data length, showing the total length of message sent. Regarding to frame check sequence (FCS), it is a checksum code using CRC-CRITT algorithm to check the data segment transmission success. User Data section contains the most important information including command and physiological signal data. In terms of wireless network protocol, MOXA device makes use of TCP/IP, IEEE 802.11 in different layers for transparent communication between serial port and WLAN nodes. However, the non-application layer sections are packaged and an application webpage is built in, where all parameters are set for transmission conversion. The exchange protocol for the interface with monitor is the universal serial standard.

Fig. 10 shows the software interface. It is composed of three modules: Parameter part, Signal Waveform part and Index part from left to right subpanel: 1) Parameter: the port number is obtained by the computer device manager. Click the start button to run for data collection if the hardware is ready, otherwise it will display error type. 2) Vital Signal Waveform: this part is synchronous with the vital signs monitor screen through the same raw physiological data exported. 3) Vital Signs Value and Index: different evaluation indexes are presented to assist doctor's surgery operation.

Moreover, updated versions record the real time data in buffer while displaying them. Every fixed time, they tag the complete case data and put them into different categories, and then call the Ethernet widget module to resend them to the server. After that, buffer is released, thus making the progress smoothly run. 


\section{Physiological Database}

The database server is established to store, manage and share the collected and analyzed data and operation details [28]. The database in charge of data, information storage and data access is based on the web browser and server model. The realization of this platform depends on the popular web server software Apache, the relational database management system MySQL, and the hypertext preprocessor PHP, all of which are widely used in server architecture [29]. Data file named by the date and operation room ID together with operation number is used to distinguish the multiple patients' information. These attributes information are stored in MySQL table and used for management. They are correlated with each waveform data file stored on specific blocks on sever disk. In database level, MySQL table design includes entity integrity, referential integrity and domain integrity like signature validation to keep the data stability, maintainability and re-usability [30]. In the entire network layer, MP60 data processing protocol and MOXA commercially qualified embedded protocol guarantees the data transmission integrity [27], which keeps the network data flow run normally and precisely. As for the signal data itself, it should be pointed out that a key step is conducted to guarantee the case data integrity. Some unexpected events may interrupt the collection procedure, therefore, the data file which contains the exact time of every data point will be verified by checking the beginning and ending time being consistent with the anesthetic records obtained from hospital staff. Besides, the number of data points recorded in the file will be compared to product of the sampling rate and recording duration.

Fig. 11 generally shows the main functions in the database. All members should be authorized to have further data access and services. The authority includes member ID check, random code verification, service acceptance and permission for safety. Users are then permitted to login, upload and download data, view, delete and further analyze by themselves. A file management system comes for the treatment. Due to different member assigned by the different authorities, the application server must identify the identity and authority of a member who requires the particular services.

\section{Results}

\subsection{Evaluation of the Data Collection System}

\subsubsection{Signal Strength of the Wireless Component}

This part adopts the situation of NTUH as example as well. To assure the quality of the network is the preliminary task of PMSDA system. Test lasts for hours over several days. All tests have similar performances and varied little. One representative test is illustrated here. As shown in Figs. 12-14, the Wifi Analyzer is employed to check the wireless performance. Wifi Analyzer has three main modes: Signal Meter, Channel Graph and Time Graph. In the signal meter mode seen in Fig. 12, the signal power strength ranges from $-100 \mathrm{dBm}$ to $-40 \mathrm{dBm}$. Different color means different strength, which means it becomes higher from grey to green. In comparison with one AP Service Set Identifier (SSID) named NTUH-Guest, which is widely used in NTUH by staffs and patients in Fig. 12(a) and acts as one referenced object here, access point of D-Link_YZU_host, which is the local gateway installed in control room to gather all data flow from different operating rooms, shows the more powerful signal strength clearly in Fig. 12(b). The repeater is marked as yzu_repeater_CR_02 as shown in Fig. 12(c), which is the SSID of the repeater to be accessed by specified MOXA NPort W2250A in one operating room. From Fig. 13, the Channel Graph mode of the Wifi Analyzer displays the channel and peak comparison among NTUH-Guest, D-Link_YZU_host and yzu_repeater_CR_02. It is obvious that peak of latter two are higher than that of NTUH-Guest. Also, from the distribution of channel, the latter two occupy the idle channel 3, which promotes the communication and quality of the wireless link by decreasing the collision with same frequency. For the stability test, as shown in Time Graph mode of Fig. 14, it plots the vibration of the signal strength. It can be roughly calculated that the average power strength of the latter two is about 1000 times stronger than NTUH-Guest.

\subsubsection{Capacity of Network Communication}

It is necessary to detect the performance of whole data acquisition part from the monitor machine in operating room to the transfer station in control room. Grasping the physiological data out from the operation room without any interrupt to the regular surgery work and successful transmission is critical even compared to the server database constructed. 
JPerf is a Java-based tool that calculates the maximum TCP and UDP without any additional hassle or complex settings. It is a frontend for Iperf [31], a popular command-line utility for bandwidth measurements with friendly user interface. Figs. 15(a-b) show the average bandwidth in TCP test mode while Figs. 16(a-b) show the delay jitter and datagram loss in UDP test mode from end to end. The results show either the UDP or TCP tests prove enough high network transmission speed to meet the data collection requirements. So do the threads tests. It lays solid foundations for multiroom automatic data transmission in terms of hardware infrastructure.

\subsubsection{Statistics of the Cases Acquired}

Preliminary statistics of performance evaluation is conducted. In order to exemplifying the capability of PMSDA, the details of surgeries operated in the specific rooms are recorded by research assistant carefully with the room number, operation date, time, operation serial number, etc. By comparing with the records, 335 intact and successful datasets were collected, which account for the majority (totally 350 cases). Table 2 gives different successful rate of each hospital operation room, and also gives the average value in the muti-hospital rooms. In comparison with manual method, an average of 3 to 5 cases are acquired per day with a worker standing by, it is obviously more efficient even through with some case loss. Since the focus is on the research goal, the data accessibility, integrity and availability, a few interrupted data cases including packet loss type or the whole case missing are just abandoned. It leads to small errors, which can be tolerated under the practical hospital environment during the age of big data mentioned in Introduction As it is generally known, one surgery usually lasts for around 3 hours consisting of three periods: preoperative, intraoperative and postoperative work, this new design saves more human resources, cost, time, etc. To sum up, the raw data makes Giga Bytes, thus creating the preliminary prototype of big physiological data for anesthesia.

\subsection{Access of physiological signal database}

Fig. 17 shows the data page of the database website, which is sponsored by National Chung-Shan Institute of Science and Technology (NCSIST), Yuan Ze University (YZU) and NUTH. It simply gives homepage for guest, who is the only role having no rights to log in, thus keeping it convenient for regular visit. Administrator can login and logout to manage database all authorities after entering the account, password and the random verification code with the highest priority and authority operation like data file management, user management, patient information management, etc. Members are allowed to download the medical signal data and upload their research achievements and ideas for sharing. The logging on service can be called by clicking the top right corner button. Point should be stressed that all the data available online in the server must be approved by the patients with their signature on the formal documents. The operation of the data must comply with research standard instead of any commercial goal or personal willing.

Besides, other general services about introduction, publication can be visited as the same way on the left side. The kernel part is the medical data pool such as ECG, EEG, BP, etc. And their corresponding patient information without any invasion of privacy based on the consent rules. As to the physiological data pool, when a visitor selects the submenu operating room date and the physiological signal, a data list will be shown on the right side of the submenu with the name, type, description and size of each dataset illustrated clearly by the pool file management system. The File Management takes charge of uploading and downloading data. However, functions like uploading, downloading and so on are operated by different users with different authority as described before, and no one can do it without the users' system permission for safety.

\subsection{DoA Research Analysis based on previous data}

For demonstration of initial reliability and feasibility of system, some previous analysis achievements are proposed here to predict DoA. General anesthesia is a reversible status change that includes unconsciousness, amnesia, analgesia, and akinesia, with concomitant stability of the cardiorespiratory and autonomic systems [32,33]. However, anesthesiologists have multiple inconsistent definitions of the anesthetic state and have no standard measurement to assess it. Therefore, many methods to detect the DoA were proposed and developed for clinical application in operating theatre, such as BIS, response entropy (RE), state entropy (SE), etc. In previous studies on DoA analysis, the entropy family was employed frequently to measure the complexity of complex time series: approximate entropy (ApEn), sample entropy (SampEn) and multiscale entropy (MSE). Additionally, empirical mode decomposition (EMD) plays an important role in decomposing and 
filtering nonlinear and nonstationary physiological data. ApEn can act as an index of degree of unconsciousness [34]. But compared to the sample entropy (SampEn), it is less sensitive to the change of complexity for online analysis [35]. In further, the distribution of complexity in different time scales is capable of describing the different states of patients during an operation by using multiscale entropy (MSE). Within these analysis papers, EMD is very useful for signal decomposition, making a clear adoption for DoA analysis [36].

Although these publication results come from the previous data, which are acquired by manual method, the principle of physiological information extraction from the export data stream of the MP60 patient monitors makes no difference. It means that substantive information is totally the same with the former theoretically. However, previous research work can, to some extent, prove the veracity of data. Further analysis based on the data collected by PMSDA system should be undertaken to demonstrate the effectiveness directly in future.

\section{Discussion}

In this PMSDA system, achievements for real-time data acquisition are made, which is acknowledged by doctors for its preliminary structure and performance. In other words, the whole wireless network system is constructed through large amounts of endeavor and dedication to practical requirements, which makes a feasible and easily convenient demonstration for some specific area research groups (e.g. Anesthesia) without any dependence and interference on HIS.

From the statistics, it is clear that the proposed system collection rate is much higher than the manual one [23], which speeds the database construction much more. Results prove the capability of data transmission and coexistence with other wireless network. Being separated with EHRs, the signal strength and coverage can be adjusted and manipulated without any conflicts with HIS based on practical requirements. This exhibits more convenient characteristic of WLAN than other shortdistance wireless methods. All above demonstrate a doable, independent, low-cost solution for medical research data collection. As to raw valid data access by visiting the database website, its experience may perform less than Cambridge Neurosurgical Unit and Harvard Physionet, including the aspects of the data category, data amount, data analysis tools, etc. It should be admitted that the database infrastructure is founded on free open source for demonstration. However, it also can promote some initial researches for all researchers, and provide a preliminary data storage and management framework.

However, this design has several limitations as well. Firstly, PMSDA is not $100 \%$ successful to collect every operation case for some reasons such as the network instability or irreverent human mistake operation. For example, our devices happened to be removed by hospital staff unintentionally during this preliminary test, resulting in the decreased system capability; however, it can be solved when the system is put in practice by professional installation. And there are also some technical reasons consisting of devices lifespan and robustness of software. Although a certain amount of errors are tolerated in big data, measures should be undertaken to eliminate the influence to the least to enhance the quality of service (QoS) of the network $[37,38]$. Secondly, system did not cover the anesthetic gas signal yet, like CO2, O2, etc. There is no doubt that these kinds of signals are necessary to gather [39]. So, improvements should be done to conquer some limitations and meet new demands. In addition, data security should become the main concern in upcoming updated design. Even though it is transferred through LAN, which already makes it much safer than Internet, it faces potential dangers when the local gateway is connected to the server. This poses us some urgent data encryption or secures monitoring improvements in case of hacking. It is necessary to enhance this inspection aspect when the server is visited. Generally speaking, this system contains no private information of patients, which somehow protects the patients under the worst conditions of data leaking. Other issues concerned the system reliability should also be taken into consideration, such as fault tolerance and self-monitoring. Deploying a self-monitoring widget not only could guarantee security quality and alarm in case of cyber invasion or abnormal data rate, but also facilitate the system regular operation through alerting signal. For example, if the website is hacked or data collection network is blocked, the corresponding lights can blink to reduce malfunction rate [40]. However, there should be some capabilities of fault tolerance to deal with some issues even though they may be detected by self-monitoring. Faults like communication errors, unstable connectivity, sensors faults may occur [41]. A software strategy like rebooting the relevant unit or designing adaptive memory cache for high volume of input data when stored into database should be developed. Schemes like priority labeling could be address to deal with some unexpected 
situations. Also, advanced distributed hardware network updates like self-organization could be undertaken to guarantee the connectivity such as MOXA access to AP [42].

In future, deepening our data mining and analysis about anesthesia filed definitely becomes our concerns. Our previous works about noise cancellation, signal reconstruction, evaluation of DoA are undertaken based on EMD and entropy families, as well as the regression and modeling methods like neural network and fuzzy logic. Recently the quasi-periodicities in EEG detected by phaserectified signal averaging propose a new insight to quantify the DoA [43,44]. And the amplitude modulation and frequency modulation (inter-mode and intra-mode) underlying the nonstationary and nonlinear data becomes a hot issue. Holo-Hilbert spectral analysis (HHSA) proposed by Norden E. Huang is used to explore these properties [45]. Interactions between frequency bands under different consciousness level induced by anesthetics arouse much interest for researches such as cross frequency coupling $[46,17]$. Besides, the advanced deep neural network applied in data analysis keeps an uprising trend, which might improve our accuracy rate of DoA evaluation $[47,48]$. In addition, another significant point is that current system only focuses on the data collection instead of real-time processing simultaneously although a standalone real-time processing demo system has been previously developed in our group [23]. Front-end user friendly interface based on the wireless system should utilize the aforementioned advanced algorithms including section 3.3 to discover new physiological patterns during surgery. Different from traditional monitors with only vital signs or raw signal, the new system aims at offering accurate index and appropriate recommendations after sophisticated computing to reduce the possible human experience bias [49,50]. These real-time processing results could provide guidance for doctors to control anesthetic procedure (potentially not limited to anesthesia) and should be capable of transmitting raw signal to the server through network, thus the system could facilitate the operations real-time and also the specific retrospective research [51]. In future, all kinds of services might be cost-effective to diagnose and treat patients if system would be ultimately upgraded to big healthcare platform with underlying big data mining and cloud computing services [52,53].

\section{Conclusion}

This paper has established a networked real-time operating physiological signal acquisition system, targeting one easy-access and low-cost standard data collection model for anesthesia, even for other specific research category in future. It generally consists of two parts: front-end collection part in operating rooms and physiological database. Based on the MP60 monitors, it potentially aims at setting a preliminary standard anesthesia data collection infrastructure under condition of little interruption to the regular work in hospital to overcome the data heterogeneity for benefits of research. For less than a month, 350 datasets are obtained, though there are some cases deficiencies during the testing time. Generally, it obviously promotes the clinical data acquisition for different kinds of patients. Also, this system design provides a data sharing server. Through the website, researcher and doctors are able to download and analyze what they are interested in. Nevertheless, further improvements and extra functions need to be undertaken to enhance the performance such as the user interface, as well as the category of clinical signs. Much work should be done to improve the collection efficiency better. Actions about the security, search function, network encryption, data mining and the application of our research results should also be conducted in future.

\section{Compliance with Ethical Standards}

Funding: This research, sponsored by Wuhan University of Technology international exchange program (Grant Number: 2015-JL-012). It was also supported by National Chung-Shan Institute of Science \& Technology in Taiwan (Grant Numbers: CSIST-095-V401) and funded by China National Nature Science Foundation (Grant Number: 51475342, 51675389).

Conflicts of Interest: The authors declare no conflict of interests with any company, funding source or an individual.

Ethical approval: As this was a wireless data collection network, system quality control, and test study, ethical approval was not required. It focuses on the system design, not any human related clinical aspects. 


\section{References}

1. Murdoch TB, Detsky AS (2013) The inevitable application of big data to health care. JAMA 309 (13):1351-1352. doi:10.1001/jama.2013.393.

2. Raghupathi W, Raghupathi V (2014) Big data analytics in healthcare: promise and potential. Health Inf Sci Syst 2:3. doi:10.1186/2047-2501-2-3.

3. Hu PF, Yang S, Li HC, Stansbury LG, Yang F, Hagegeorge G, Miller C, Rock P, Stein DM, Mackenzie CF (2017) Reliable Collection of Real-Time Patient Physiologic Data from less Reliable Networks: a "Monitor of Monitors" System (MoMs). J Med Syst 41 (1):3. doi:10.1007/s10916-016-0648-5.

4. Li J-S, Zhang Y-F, Tian Y (2016) Medical Big Data Analysis in Hospital Information System. In: Soto SV, Luna JM, Cano A (eds) Big Data on Real-World Applications. InTech, Rijeka, p Ch. 04. doi:10.5772/63754.

5. White T (2012) Hadoop: The definitive guide. " O'Reilly Media, Inc.",

6. Zaharia M, Chowdhury M, Franklin MJ, Shenker S, Stoica I (2010) Spark: Cluster Computing with Working Sets. HotCloud 10 (10-10):95.

7. Iqbal MH, Soomro TR (2015) Big data analysis: Apache storm perspective. International Journal of Computer Trends and Technology:9-14.

8. Schmidt PE, Meredith P, Prytherch DR, Watson D, Watson V, Killen RM, Greengross P, Mohammed MA, Smith GB (2015) Impact of introducing an electronic physiological surveillance system on hospital mortality. BMJ Qual Saf 24 (2):176-177. doi:10.1136/bmjqs2014-003845.

9. Nguyen L, Bellucci E, Nguyen LT (2014) Electronic health records implementation: an evaluation of information system impact and contingency factors. Int J Med Inform 83 (11):779-796. doi:10.1016/j.ijmedinf.2014.06.011.

10. Kruse CS, Kristof C, Jones B, Mitchell E, Martinez A (2016) Barriers to electronic health record adoption: a systematic literature review. Journal of medical systems 40 (12):252.

11. Menachemi N, Collum TH (2011) Benefits and drawbacks of electronic health record systems. Risk Manag Healthc Policy 4:47-55.

12. . http://www.physionet.org/.

13. Goldberger AL (2000) Components of a New Research Resource for Complex Physiologic Signals, PhysioBank, PhysioToolkit, and PhysioNet, American Heart Association Journals. Circulation 101 (23):1-9.

14. a. http://www.neurosurg.cam.ac.uk/pages/ICM/.

15. Brown EN, Lydic R, Schiff ND (2010) General anesthesia, sleep, and coma. N Engl J Med 363 (27):2638-2650. doi:10.1056/NEJMra0808281.

16. Hudetz AG, Mashour GA (2016) Disconnecting Consciousness: Is There a Common Anesthetic End Point? Anesth Analg 123 (5):1228-1240. doi:10.1213/ANE.0000000000001353.

17. Mukamel EA, Pirondini E, Babadi B, Wong KF, Pierce ET, Harrell PG, Walsh JL, SalazarGomez AF, Cash SS, Eskandar EN, Weiner VS, Brown EN, Purdon PL (2014) A transition in brain state during propofol-induced unconsciousness. J Neurosci 34 (3):839-845. doi:10.1523/JNEUROSCI.5813-12.2014.

18. Pal D, Silverstein BH, Lee H, Mashour GA (2016) Neural Correlates of Wakefulness, Sleep, and General Anesthesia: An Experimental Study in Rat. Anesthesiology 125 (5):929-942. doi:10.1097/ALN.0000000000001342.

19. Yanagawa T, Chao ZC, Hasegawa N, Fujii N (2013) Large-scale information flow in conscious and unconscious states: an ECoG study in monkeys. PLoS One 8 (11):e80845. doi:10.1371/journal.pone.0080845.

20. Zakim D, Schwab M (2015) Data collection as a barrier to personalized medicine. Trends in pharmacological sciences 36 (2):68-71.

21. Greenhalgh T, Howick J, Maskrey N, Evidence Based Medicine Renaissance G (2014) Evidence based medicine: a movement in crisis? BMJ 348:g3725. doi:10.1136/bmj.g3725

22. Zilberberg MD (2011) The clinical research enterprise: time to change course? JAMA 305 (6):604-605. doi:10.1001/jama.2011.104.

23. Wei Q, Li Y, Fan SZ, Liu Q, Abbod MF, Lu CW, Lin TY, Jen KK, Wu SJ, Shieh JS (2014) A critical care monitoring system for depth of anaesthesia analysis based on entropy analysis and physiological information database. Australas Phys Eng Sci Med 37 (3):591-605. doi:10.1007/s13246-014-0285-6.

24. Yuce MR (2010) Implementation of wireless body area networks for healthcare systems. Sensors and Actuators A: Physical 162 (1):116-129. 
25. Ding Y, Hong SH, Lu R, Kim J, Lee YH, Xu A, Xiaobing L Experimental investigation of the packet loss rate of wireless industrial networks in real industrial environments. In: Information and Automation, 2015 IEEE International Conference on, 2015. IEEE, pp 1048-1053.

26. Yuce MR, Keong HC, Chae MS (2009) Wideband communication for implantable and wearable systems. IEEE transactions on microwave theory and techniques 57 (10):2597-2604.

27. http://www.moxa.com/product/NPort_W2150A_W2250A.htm.

28. Koelstra S, Muhl C, Soleymani M, Lee J-S, Yazdani A, Ebrahimi T, Pun T, Nijholt A, Patras I (2012) Deap: A database for emotion analysis; using physiological signals. IEEE Transactions on Affective Computing 3 (1):18-31.

29. Glass MK, Le Scouarnec Y, Naramore E, Mailer G, Stolz J, Gerner J (2004) Beginning PHP, Apache, MySQL Web Development. John Wiley \& Sons.

30. Imran M, Hlavacs H, Haq IU, Jan B, Khan FA, Ahmad A (2017) Provenance based data integrity checking and verification in cloud environments. PloS one 12 (5):e0177576

31. Tirumala A, Qin F, Dugan J, Ferguson J, Gibbs K (2005) Iperf: The TCP/UDP bandwidth measurement tool. htt p://dast nlanr net/Projects.

32. Akeju O, Song AH, Hamilos AE, Pavone KJ, Flores FJ, Brown EN, Purdon PL (2016) Electroencephalogram signatures of ketamine anesthesia-induced unconsciousness. Clin Neurophysiol 127 (6):2414-2422. doi:10.1016/j.clinph.2016.03.005.

33. Changeux JP (2012) Conscious processing: implications for general anesthesia. Curr Opin Anaesthesiol 25 (4):397-404. doi:10.1097/ACO.0b013e32835561de.

34. Fan S-Z, Yeh J-R, Chen B-C, Shieh J-S (2011) Comparison of EEG approximate entropy and complexity measures of depth of anaesthesia during inhalational general anaesthesia. Journal of Medical and Biological Engineering 31 (5):359-366.

35. Wei Q, Liu Q, Fan S-Z, Lu C-W, Lin T-Y, Abbod MF, Shieh J-S (2013) Analysis of EEG via multivariate empirical mode decomposition for depth of anesthesia based on sample entropy. Entropy 15 (9):3458-3470.

36. Liu Q, Wei Q, Fan S-Z, Lu C-W, Lin T-Y, Abbod MF, Shieh J-S (2012) Adaptive computation of multiscale entropy and its application in EEG signals for monitoring depth of anesthesia during surgery. Entropy 14 (6):978-992.

37. Rolim CO, Koch FL, Westphall CB, Werner J, Fracalossi A, Salvador GS A cloud computing solution for patient's data collection in health care institutions. In: eHealth, Telemedicine, and Social Medicine, 2010. ETELEMED'10. Second International Conference on, 2010. IEEE, pp 95-99.

38. Mahapatra A, Anand K, Agrawal DP (2006) QoS and energy aware routing for real-time traffic in wireless sensor networks. Computer Communications 29 (4):437-445.

39. Patel MG, Swadia V (2017) Role of EtCO2 (End tidal CO2) Monitoring (Capnography) During Laparoscopic Surgery under General Anesthesia. Int J Res Med 5 (4):148-154

40. Hsin C, Liu M (2006) Self-monitoring of wireless sensor networks. computer communications 29 (4):462-476.

41. Bhuiyan MZA, Wang G, Cao J, Wu J (2015) Deploying wireless sensor networks with faulttolerance for structural health monitoring. IEEE Transactions on Computers 64 (2):382-395.

42. Koushanfar F, Potkonjak M, Sangiovanni-Vincentell A Fault tolerance techniques for wireless ad hoc sensor networks. In: Sensors, 2002. Proceedings of IEEE, 2002. IEEE, pp 1491-1496.

43. Liu Q, Chen Y-F, Fan S-Z, Abbod M, Shieh J-S (2017) Quasi-Periodicities Detection Using Phase-Rectified Signal Averaging in EEG Signals as a Depth of Anesthesia Monitor. IEEE Transactions on Neural Systems and Rehabilitation Engineering.

44. Liu Q, Chen Y-F, Fan S-Z, Abbod MF, Shieh J-S (2016) Improved spectrum analysis in EEG for measure of depth of anesthesia based on phase-rectified signal averaging. Physiological Measurement 38 (2):116.

45. Huang NE, Hu K, Yang AC, Chang H-C, Jia D, Liang W-K, Yeh JR, Kao C-L, Juan C-H, Peng CK (2016) On Holo-Hilbert spectral analysis: a full informational spectral representation for nonlinear and non-stationary data. Phil Trans R Soc A 374 (2065):20150206.

46. Blain-Moraes S, Lee U, Ku S, Noh G, Mashour GA (2014) Electroencephalographic effects of ketamine on power, cross-frequency coupling, and connectivity in the alpha bandwidth. Frontiers in systems neuroscience 8:114.

47. Nickerson P, Tighe P, Shickel B, Rashidi P Deep neural network architectures for forecasting analgesic response. In: Engineering in Medicine and Biology Society (EMBC), 2016 IEEE 38th Annual International Conference of the, 2016. IEEE, pp 2966-2969.

48. Silver D, Huang A, Maddison CJ, Guez A, Sifre L, Van Den Driessche G, Schrittwieser J, Antonoglou I, Panneershelvam V, Lanctot M (2016) Mastering the game of Go with deep neural networks and tree search. Nature 529 (7587):484-489. 
49. Chen D, Li D, Xiong M, Bao H, Li X (2010) GPGPU-aided ensemble empirical-mode decomposition for EEG analysis during anesthesia. IEEE Transactions on Information Technology in Biomedicine 14 (6):1417-1427.

50. Zandi AS, Javidan M, Dumont GA, Tafreshi R (2010) Automated real-time epileptic seizure detection in scalp EEG recordings using an algorithm based on wavelet packet transform. IEEE Transactions on Biomedical Engineering 57 (7):1639-1651.

51. Wanderer JP, Sandberg WS, Ehrenfeld JM (2011) Real-time alerts and reminders using information systems. Anesthesiology clinics 29 (3):389-396.

52. Raghupathi W, Raghupathi V (2014) Big data analytics in healthcare: promise and potential. Health information science and systems 2 (1):3.

53. Zhang Y, Qiu M, Tsai C-W, Hassan MM, Alamri A (2017) Health-CPS: Healthcare cyberphysical system assisted by cloud and big data. IEEE Systems Journal 11 (1):88-95. 
Table 1 Wireless Technology Used in Medical Monitoring.

WMTS= Wireless Medical Telemetry Service; UWB=Ultra-Wideband; ZB = Zigbee; BT = Bluetooth; NFC= Near Field Communication; WLAN = Wireless Local Area Network.

\begin{tabular}{|c|c|c|c|c|c|c|}
\hline & WMTS & $\begin{array}{l}\text { UWB } \\
\text { IEEE } \\
802.15 .6\end{array}$ & $\begin{array}{l}\text { IEEE } \\
802.15 .4 \\
\text { (ZigBee) }\end{array}$ & $\begin{array}{l}\text { IEEE } \\
802.15 .1 \\
\text { (Bluetooth) }\end{array}$ & NFC & $\begin{array}{l}\text { WLAN } \\
(802.11 a / b / g)\end{array}$ \\
\hline Frequency & 608-614 MHz, & & & & & \\
\hline Band & $\begin{array}{l}1395-1400 \mathrm{MHz} \\
1429-1432 \mathrm{MHz}\end{array}$ & 3-10 GHz & $2.4 \mathrm{GHz}$ & $2.4 \mathrm{GHz}$ & $2.4 \mathrm{GHz}$ & $2.4 \mathrm{GHz}$ \\
\hline Bandwidth & $6 \mathrm{MHz}$ & $>500 \mathrm{MHz}$ & $5 \mathrm{MHz}$ & $1 \mathrm{MHz}$ & $1.8 \mathrm{MHz}$ & $20 \mathrm{MHz}$ \\
\hline Data Rate & 76 kbps & 850kbps 20 Mbps & 250 kbps & 721 kbps & $424 \mathrm{Kbps}$ & >11Mbps \\
\hline Range & $>100 \mathrm{~m}$ & $1-2 \mathrm{~m}$ & $0 \sim 10 \mathrm{~m}$ & $10-100 \mathrm{~m}$ & $10 \mathrm{~cm}$ & $0 \sim 100 \mathrm{~m}$ \\
\hline
\end{tabular}

Table 2 The Summary of Case Collection Results IN Both NTUH and NTUCH, Respectively.

(a) The Data Acquiring Information of 3 Operation Rooms in NTUH: I, II, III With Successful Rate of 94.49\%, 93.65\%, 95.83\% and Average Value 93.28\%; (b) The Data Acquiring Information of 3 Operation Rooms in NTUCH: IV, V, VI With Successful Rate of 98.60\%, 95.94\%, 97.11\% and Average Value 97.22\%.

\begin{tabular}{ccccc}
\hline \multicolumn{5}{c}{ (a) } \\
NTUH & Days & Sum & Success & Rate \\
\hline room I & 18 & 47 & 43 & $91.49 \%$ \\
room II & 23 & 63 & 59 & $93.65 \%$ \\
room III & 8 & 24 & 23 & $95.83 \%$ \\
\hline Total & & 134 & 125 & $93.28 \%$ \\
\hline \hline
\end{tabular}

\begin{tabular}{ccccc}
\multicolumn{5}{c}{ (b) } \\
\hline \hline NTUCH & Days & Sum & Success & Rate \\
\hline room IV & 19 & 73 & 72 & $98.60 \%$ \\
room V & 23 & 74 & 71 & $95.94 \%$ \\
room VI & 23 & 69 & 67 & $97.11 \%$ \\
\hline Total & & 216 & 210 & $97.22 \%$ \\
\hline \hline
\end{tabular}




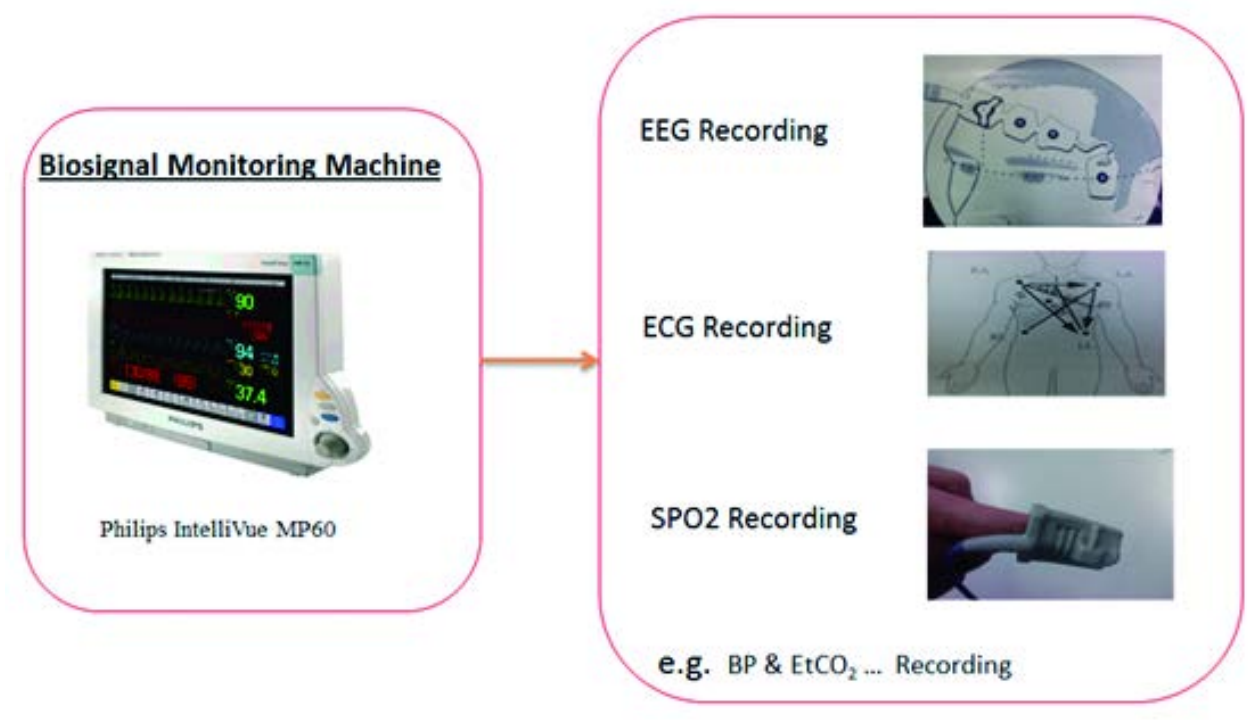

Fig. 1 Brief overview of the monitor function. It provides the EEG, ECG, PPG (SPO2), BP, RESP medical signal for research. The demo gives an overall understanding of signal category. $\mathrm{EEG}=$ electroencephalogram; $\mathrm{ECG}=$ electrocardiograph; $\mathrm{PPG}=$ photoplethysmogram; RESP=Respiration; EtCO2= End-tidal carbon dioxide tension. 


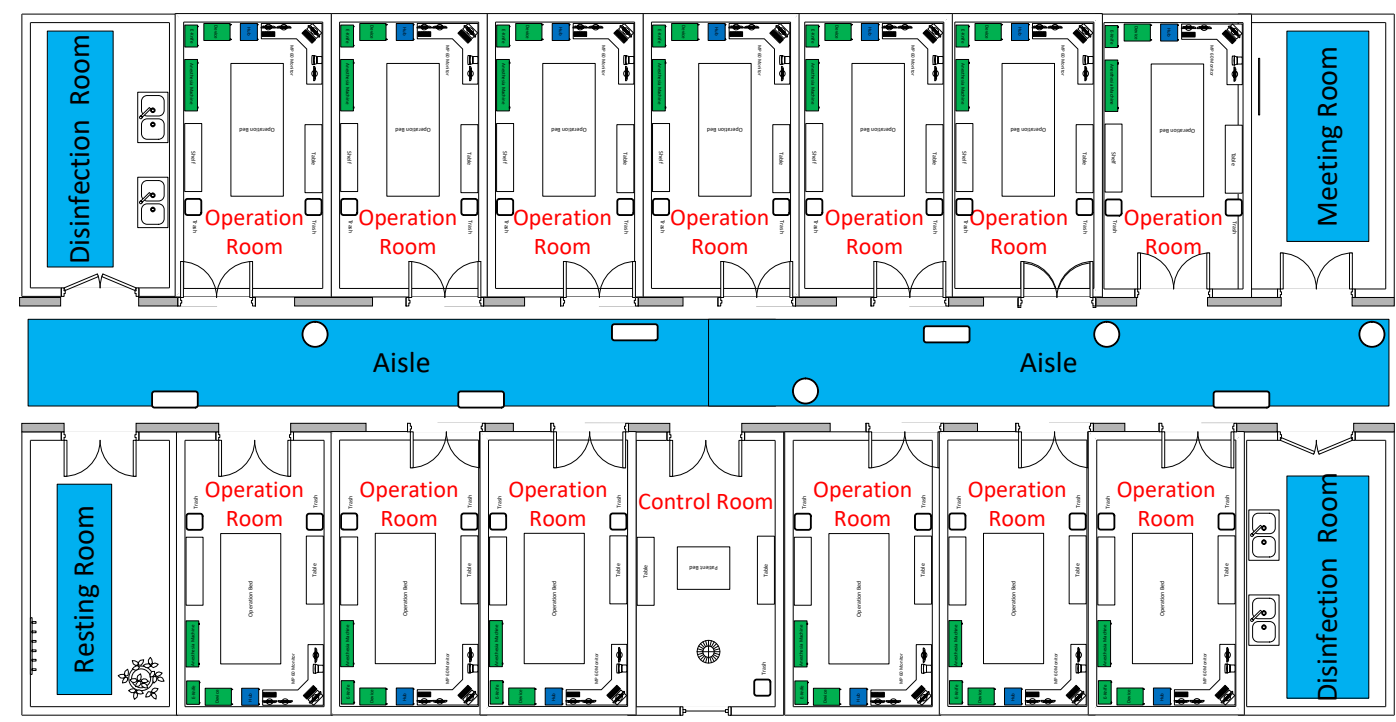

Fig. 2 General layout of the hospital operation building floors. The limited space should be separated into different areas for operation, disinfection, storage, resting, etc. Space distribution was taken into consideration before designing the whole system.

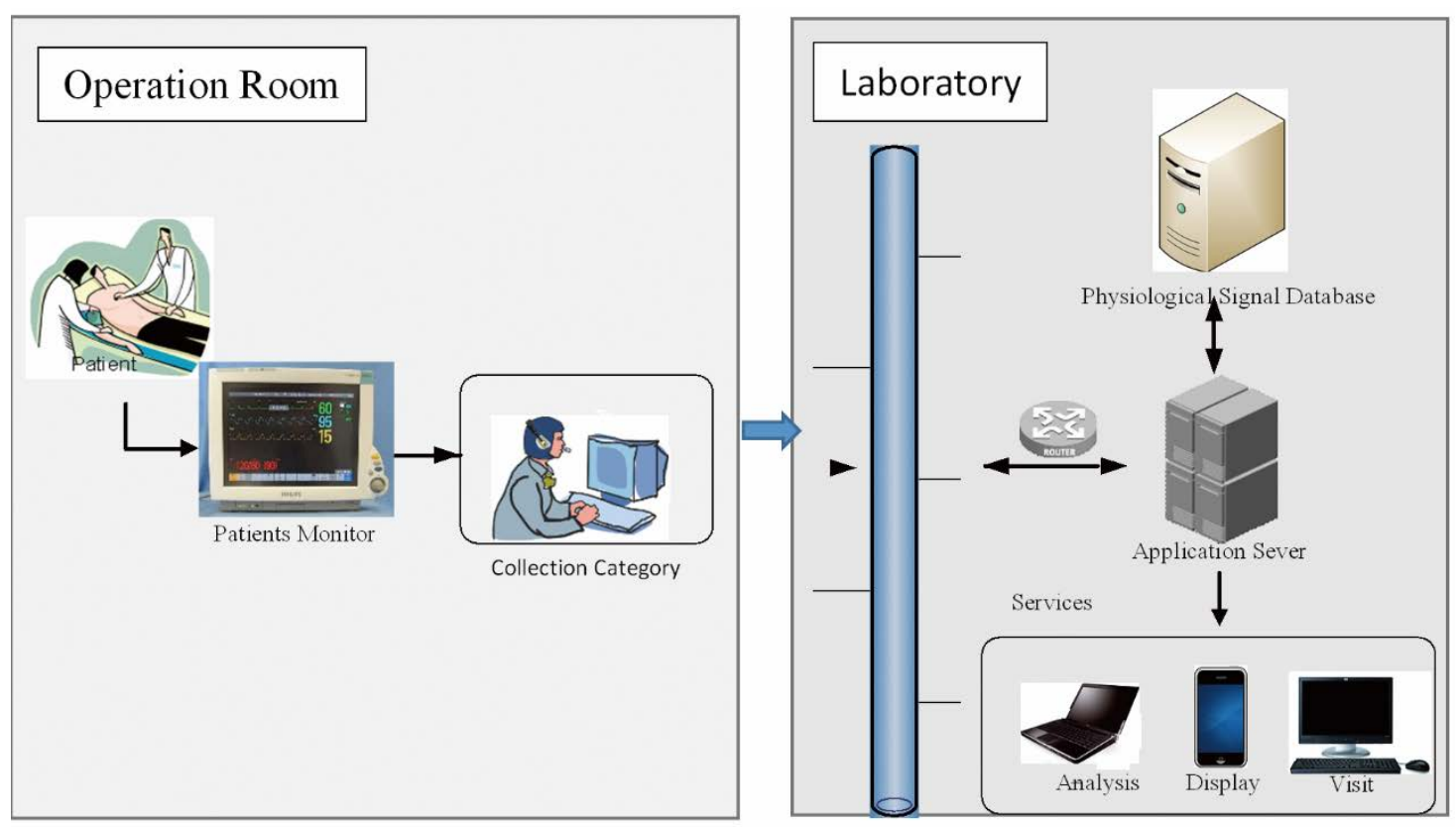

Fig. 3 The original standalone structure of data collection system. 


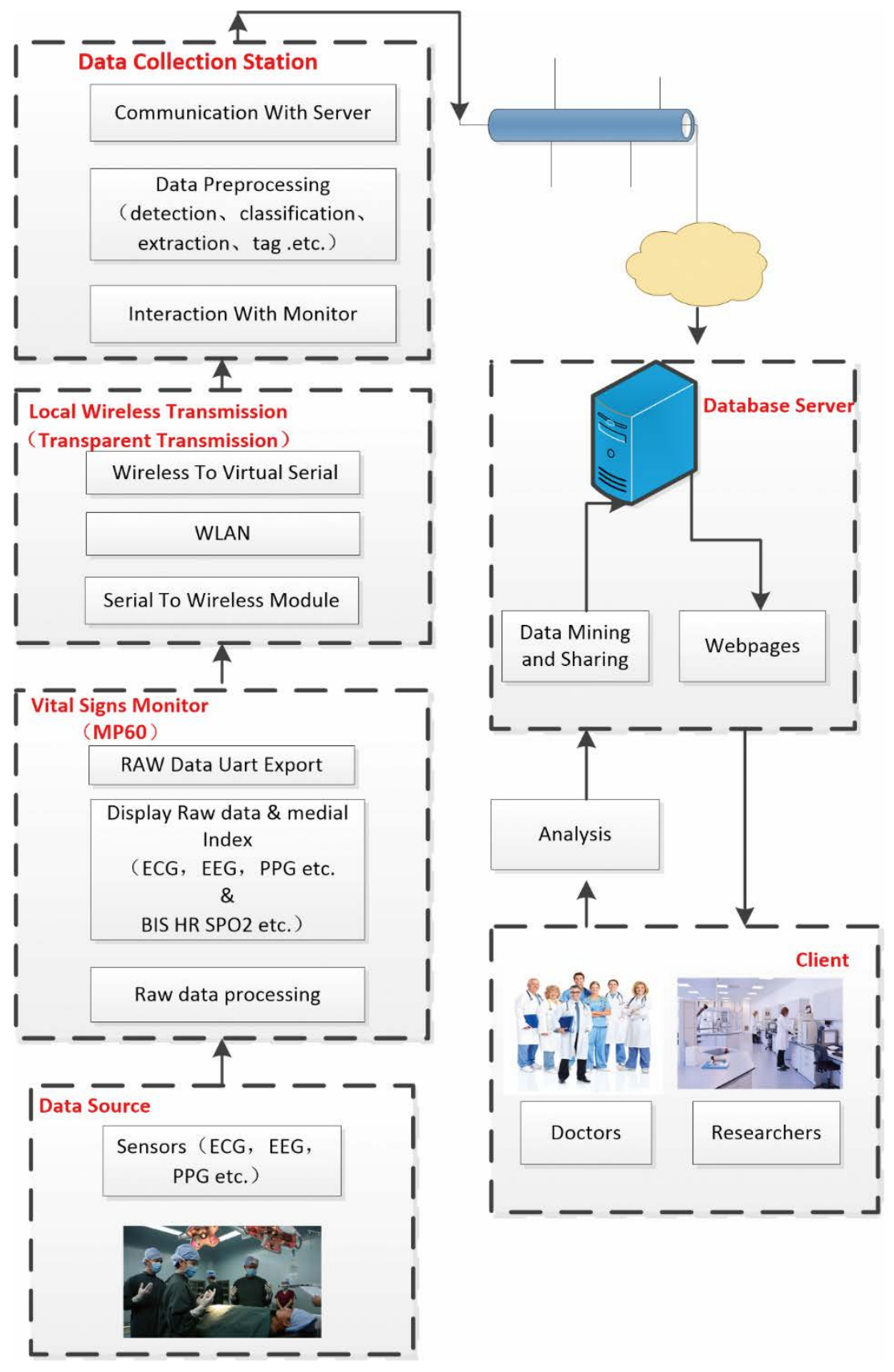

Fig. 4 Framework of the system. 


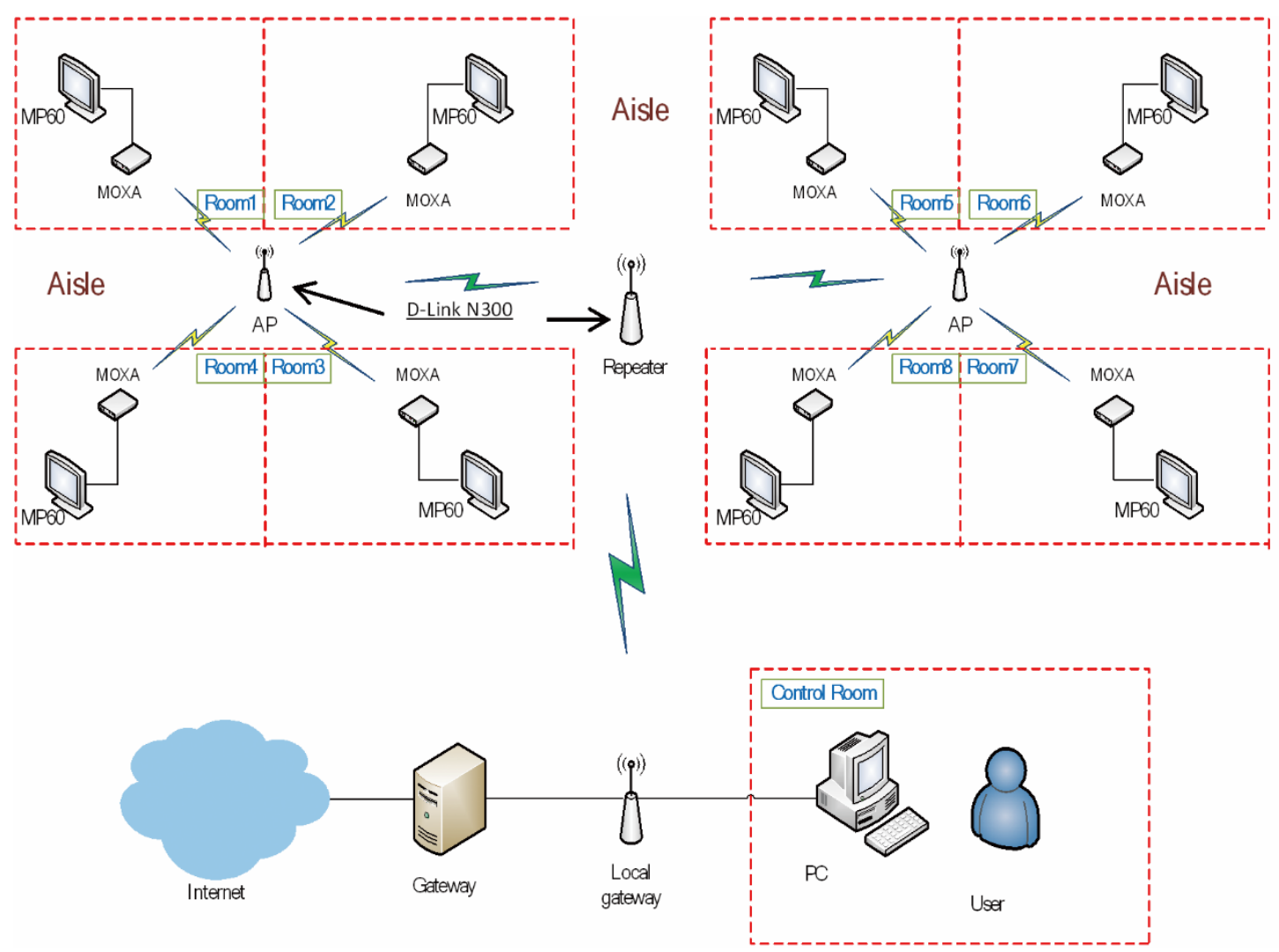

Fig. 5 Hardware of the data collection system.

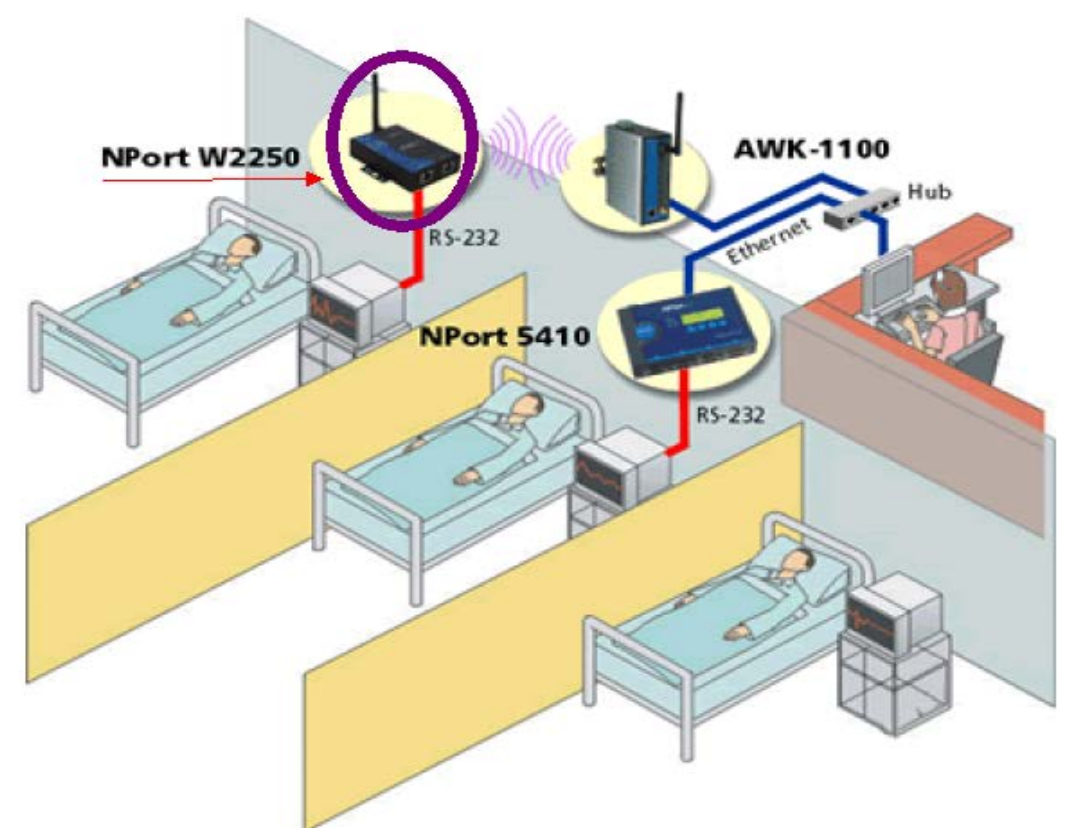

Fig. 6 A demo application of NPort W2250 [27] (used with permission). 


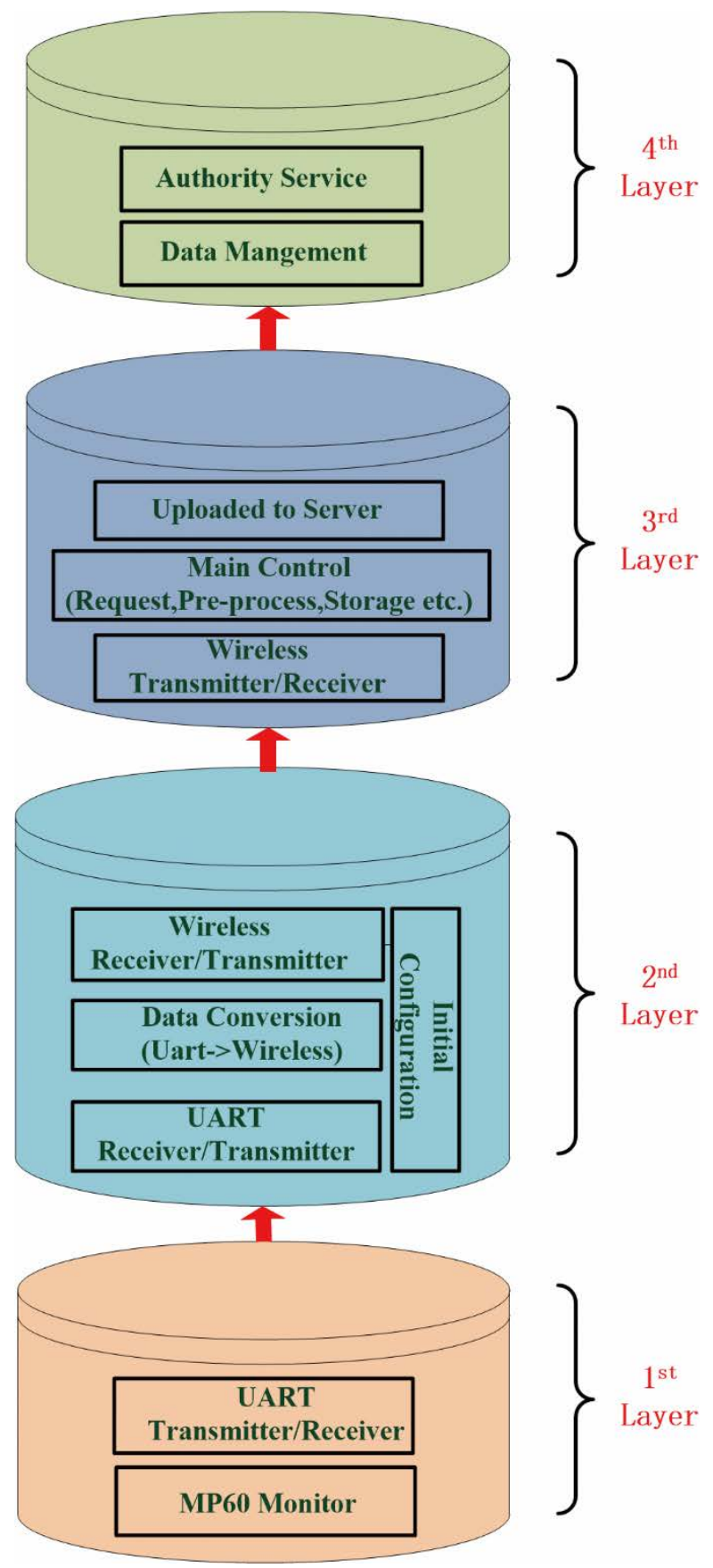

Fig. 7 Data export protocol dialog. 


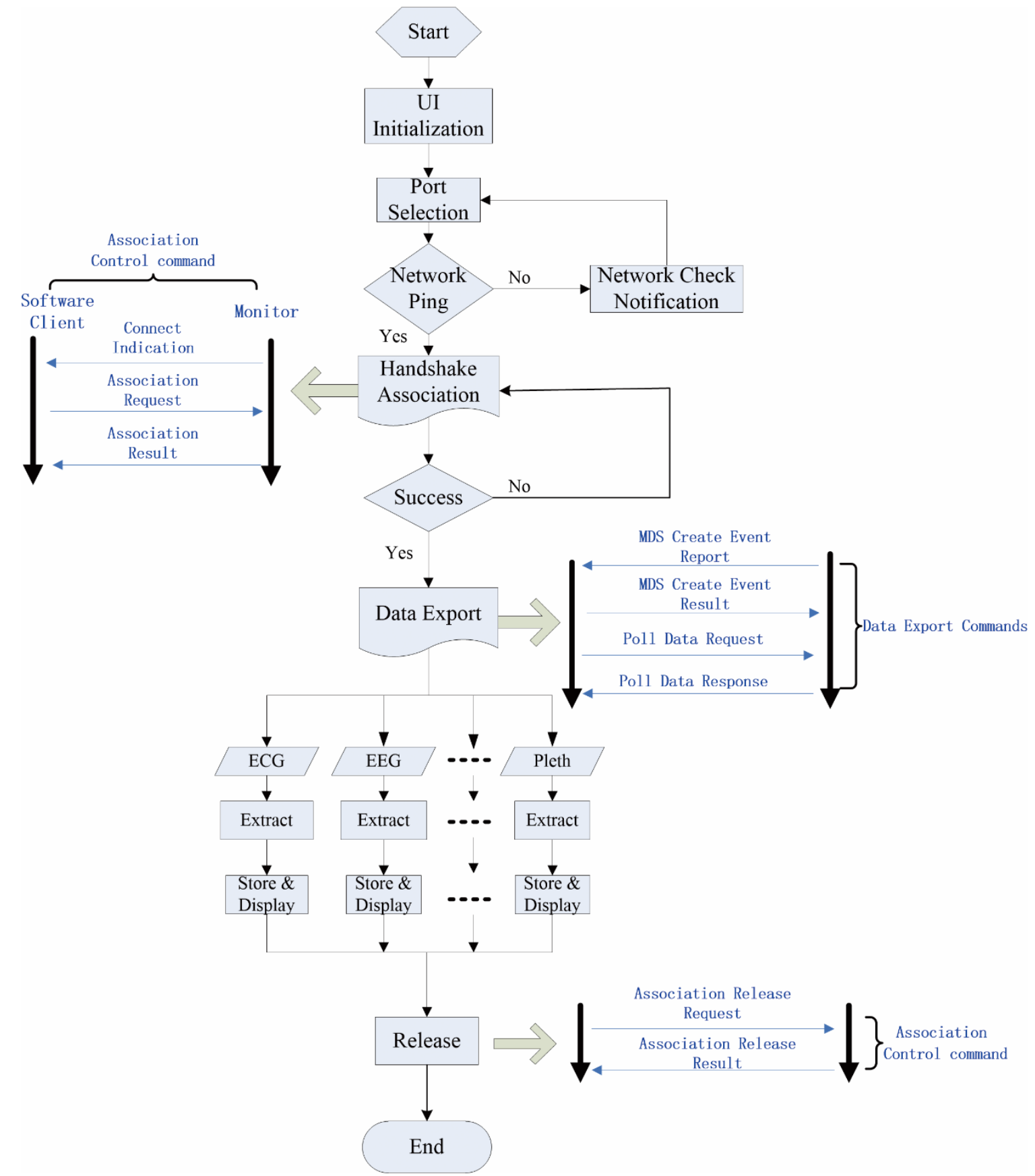

Fig. 8 Monitor data release flowchart 


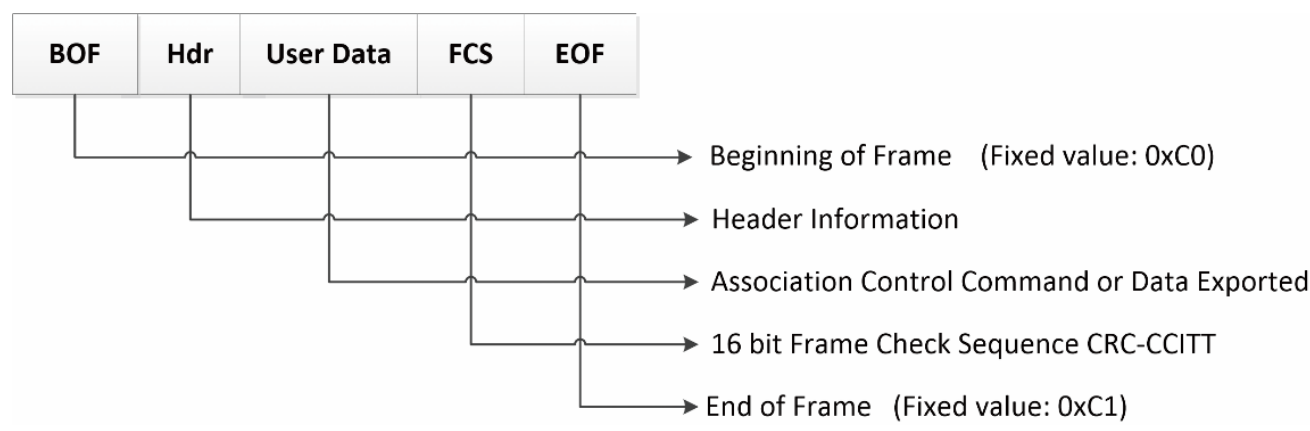

Fig. 9 Data packet format

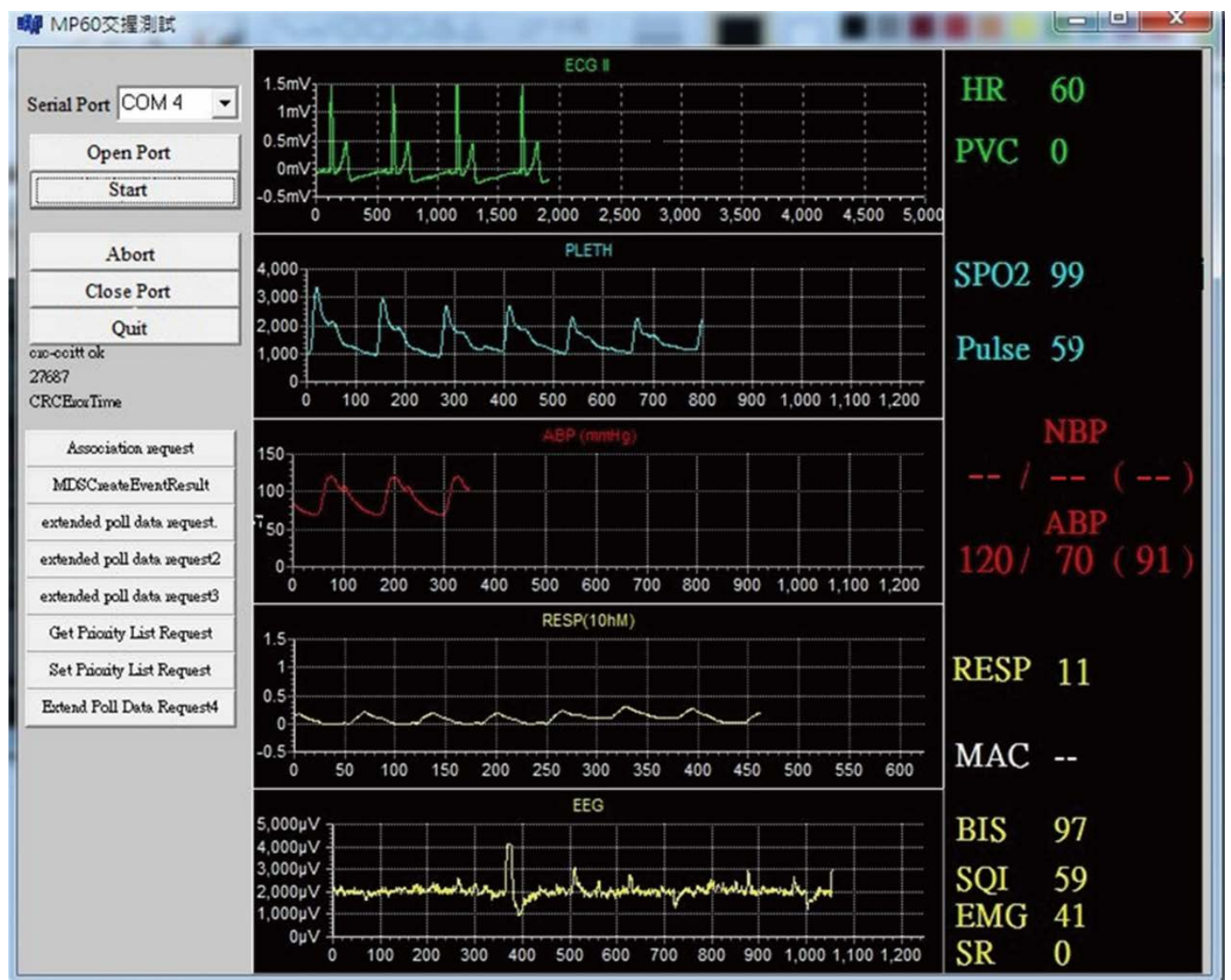

Fig. 10 Collection software of MP60 with awake status of an ordinary patient. Left panel shows the parameters and action option. Middle part is the raw signal plotting window, the data is recorded simultaneously. Right panel presents the basic vital signs of human's physical condition. HR: heart rate; PVC: Premature Ventricular Contraction; NBP: Non-invasive Blood Pressure (systolic pressure/ diastolic pressure/ mean pressure); RESP: Respiration; MAC: Minimum Alveolar Concentration; BIS: Bispectral Index with range scales: awake (80 100); induction with little response to loud audio (60 80); general anesthesia (40 60); deep anesthesia (40 60); burst suppression (0 20); SQI: Signal Quality Index; EMG: Electromyography; SR: Suppression Ratio. 


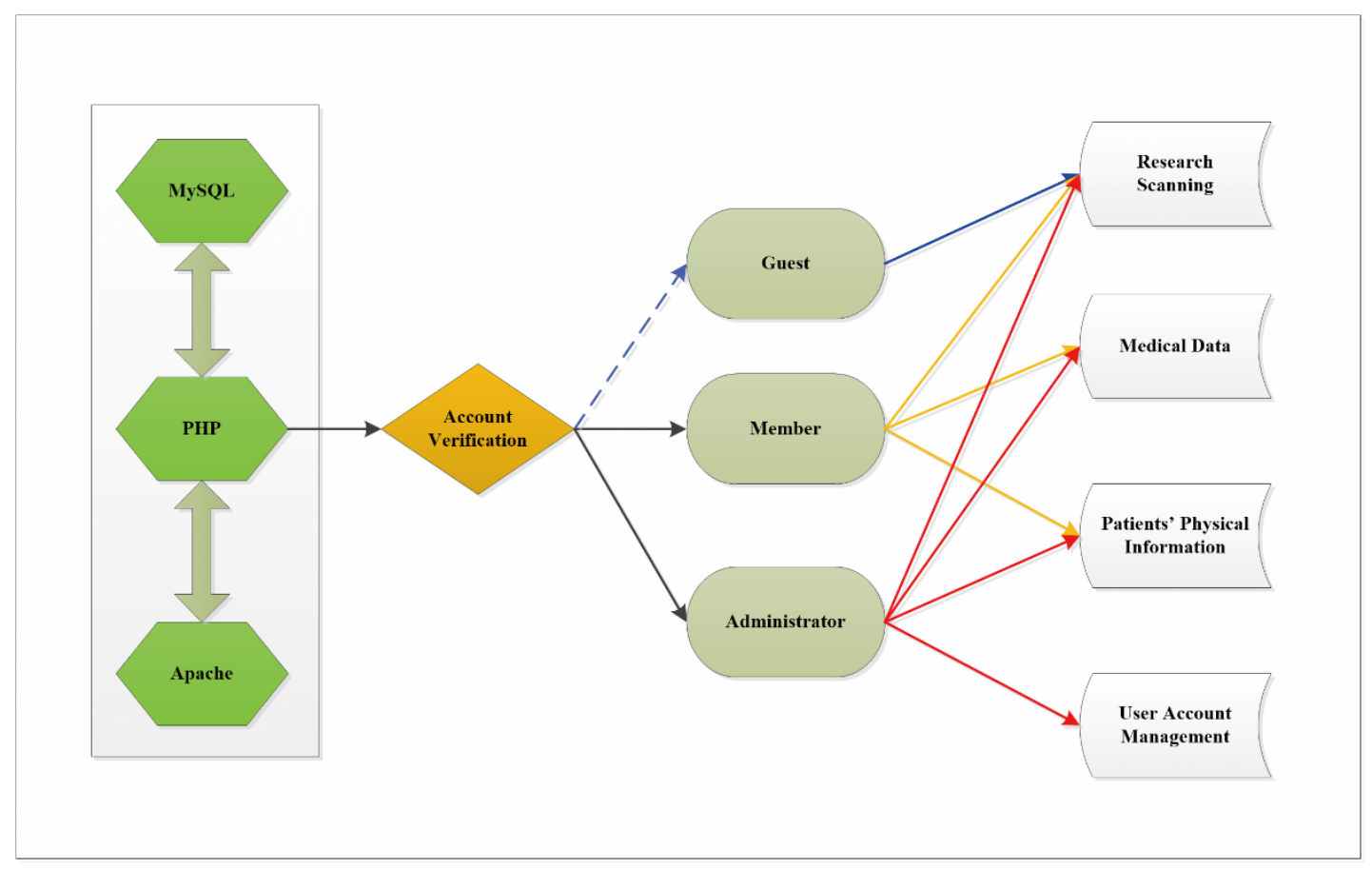

Fig. 11 The functional block chart of database.

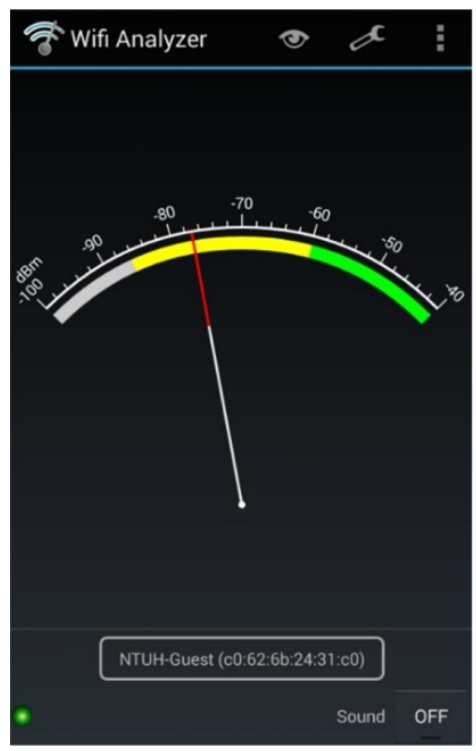

(a)

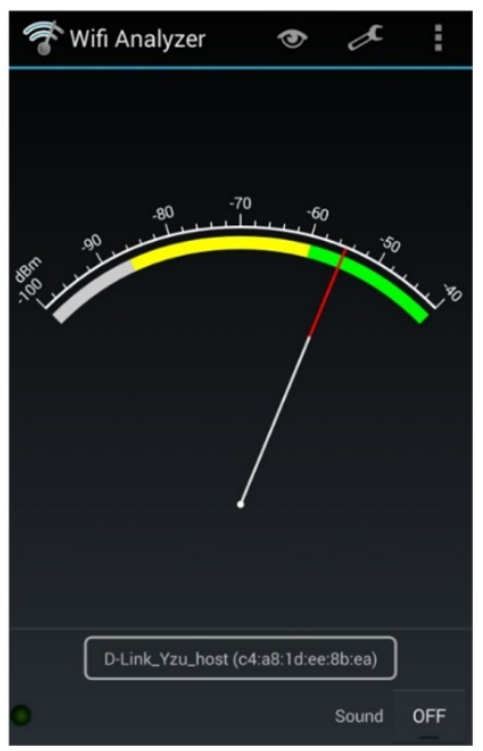

(b)

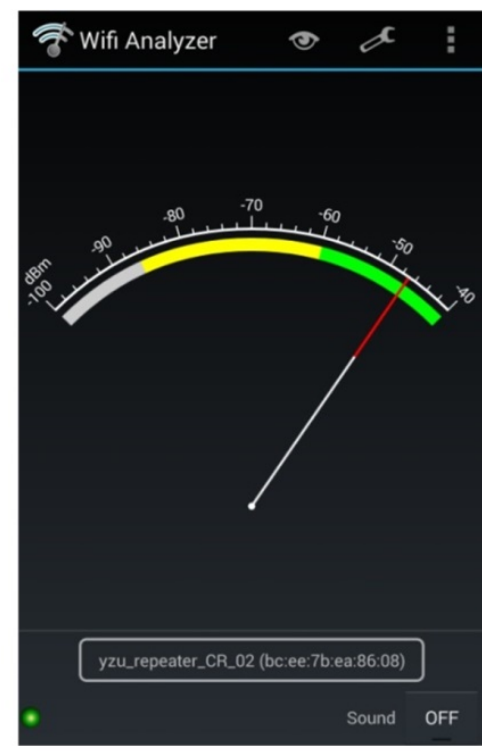

(c)

Fig. 12 Signal meter mode. The signal power strength ranges from $-100 \mathrm{dBm}$ to $-40 \mathrm{dBm}$. Different color means different strength, which means it becomes higher from grey to green.( a) The signal power strength of Service Set Identifier (SSID) named NTUH-Guest access point, which is widely used in NTUH by staffs and patients and acts as one referenced object here with others. (b) In comparison with NTUH-Guest, access point of D-Link_YZU_host, which is the local gateway installed in administrative room to gather all data flow from different operating rooms, shows the more powerful signal strength clearly. (c) The repeater is marked as yzu_repeater_CR_02, which is the SSID of the repeater to be accessed by specified Moxa NPort W2250A in one operating room, weaker than YZU_host, much stronger than NTUH-Guest. 


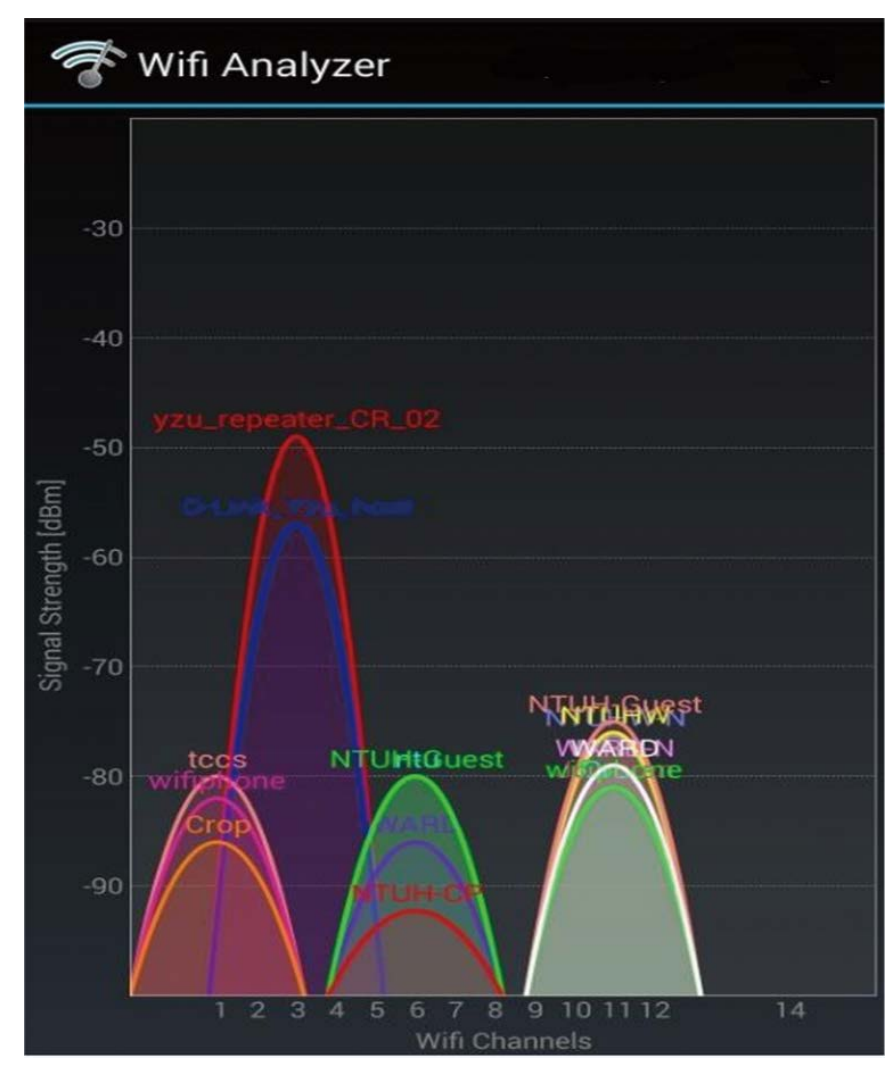

Fig. 13 Channel graph mode.

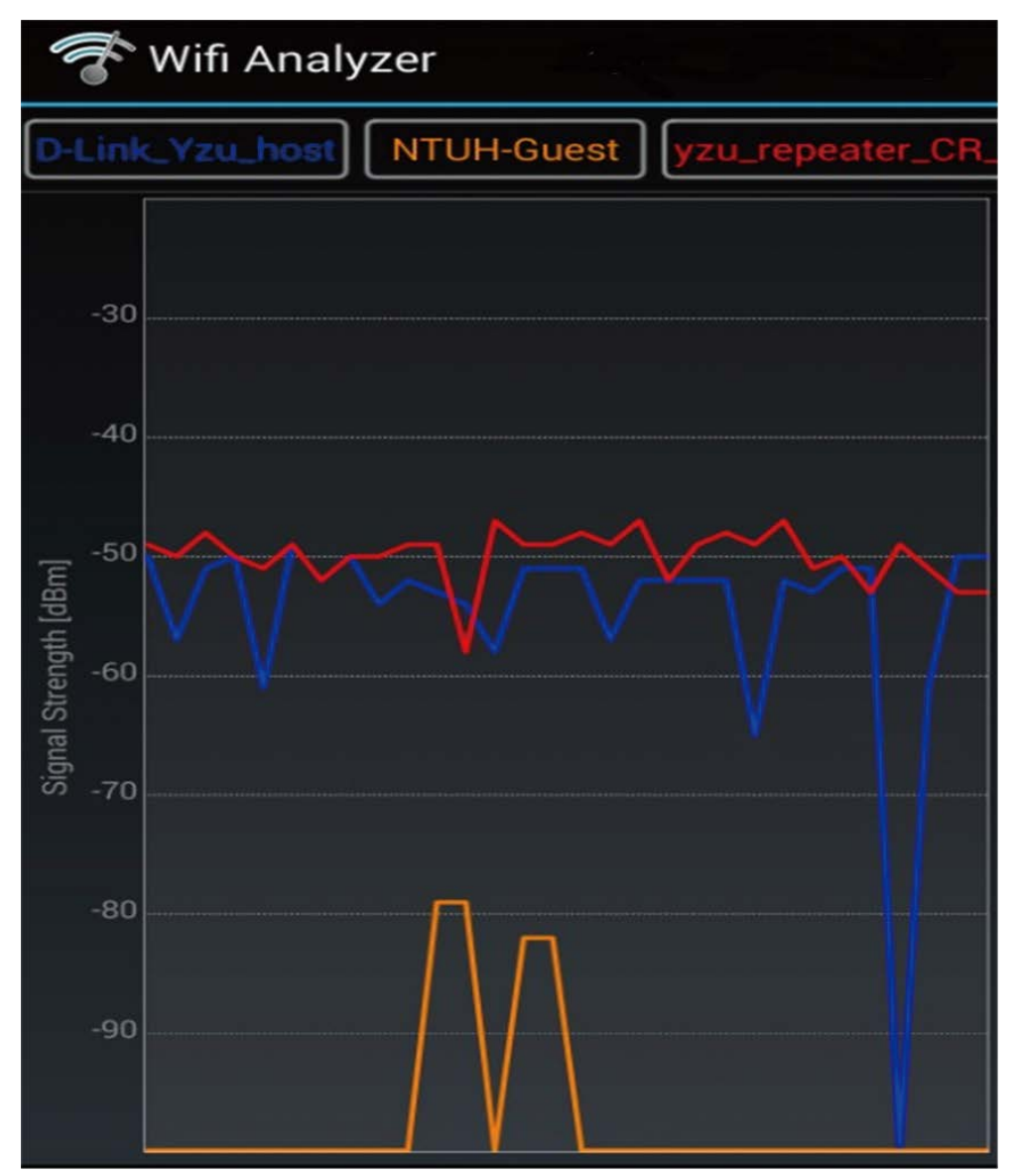

Fig. 14 Time graph mode: different color means different access point. 


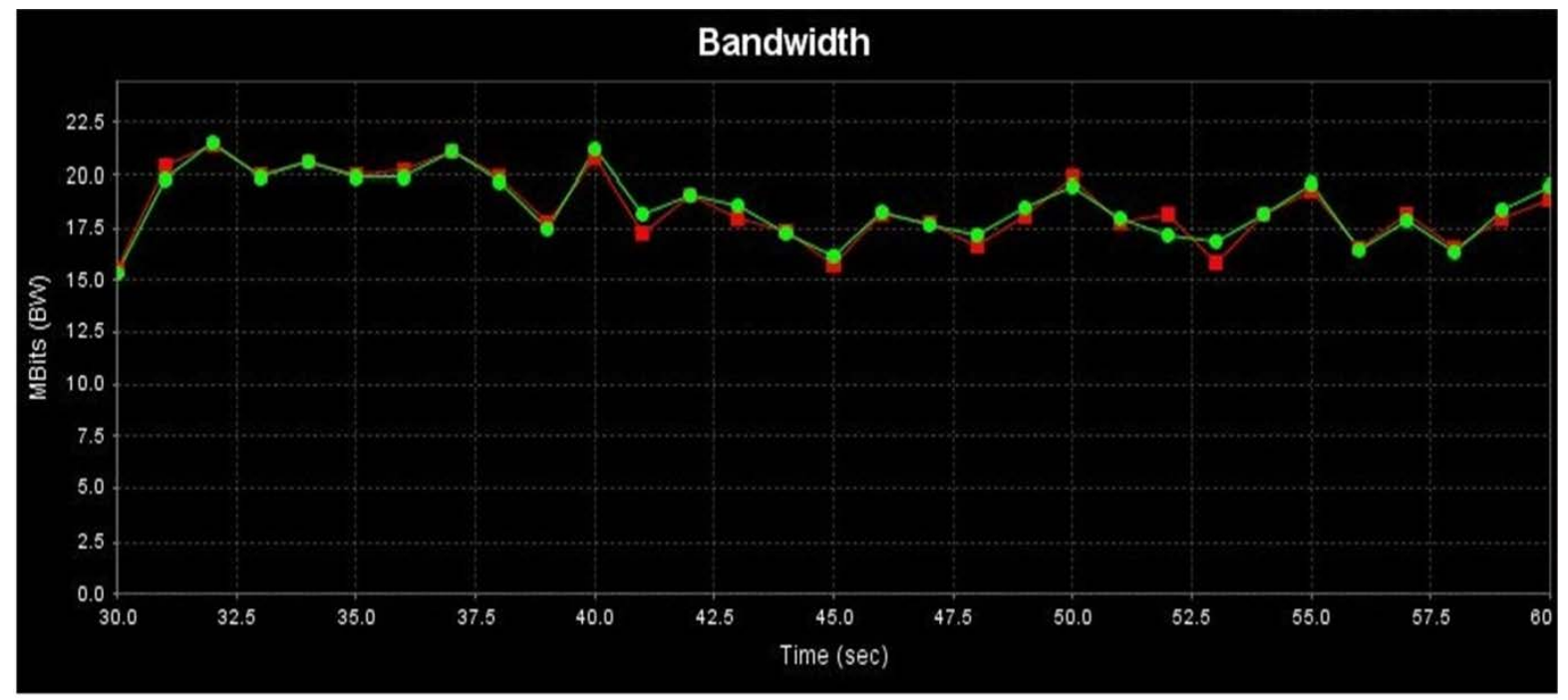

(a)

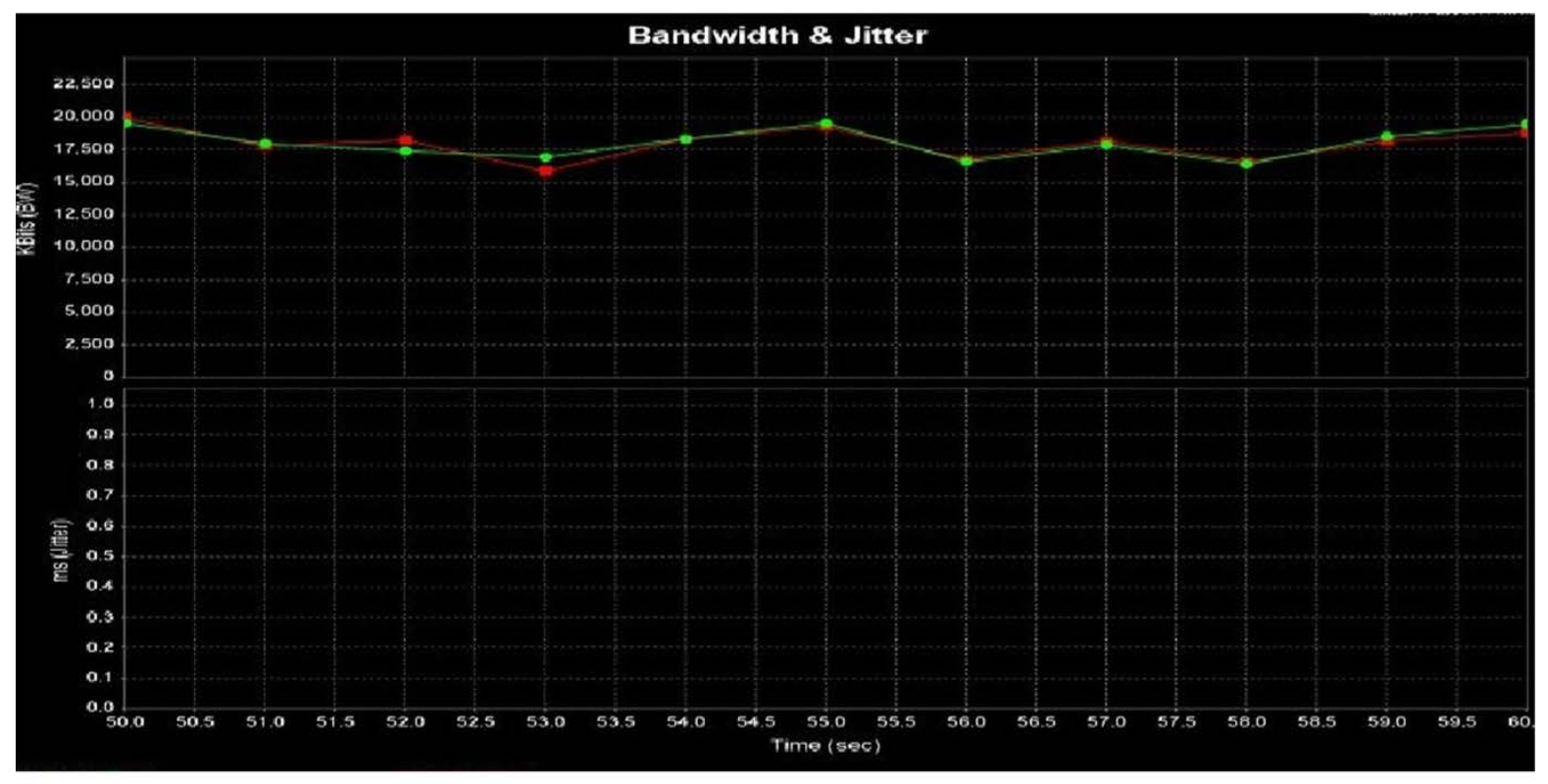

(b)

Fig. 15 Link test for Bandwidth TCP in 2 threads: (a) Client interface, (b) Server interface. 


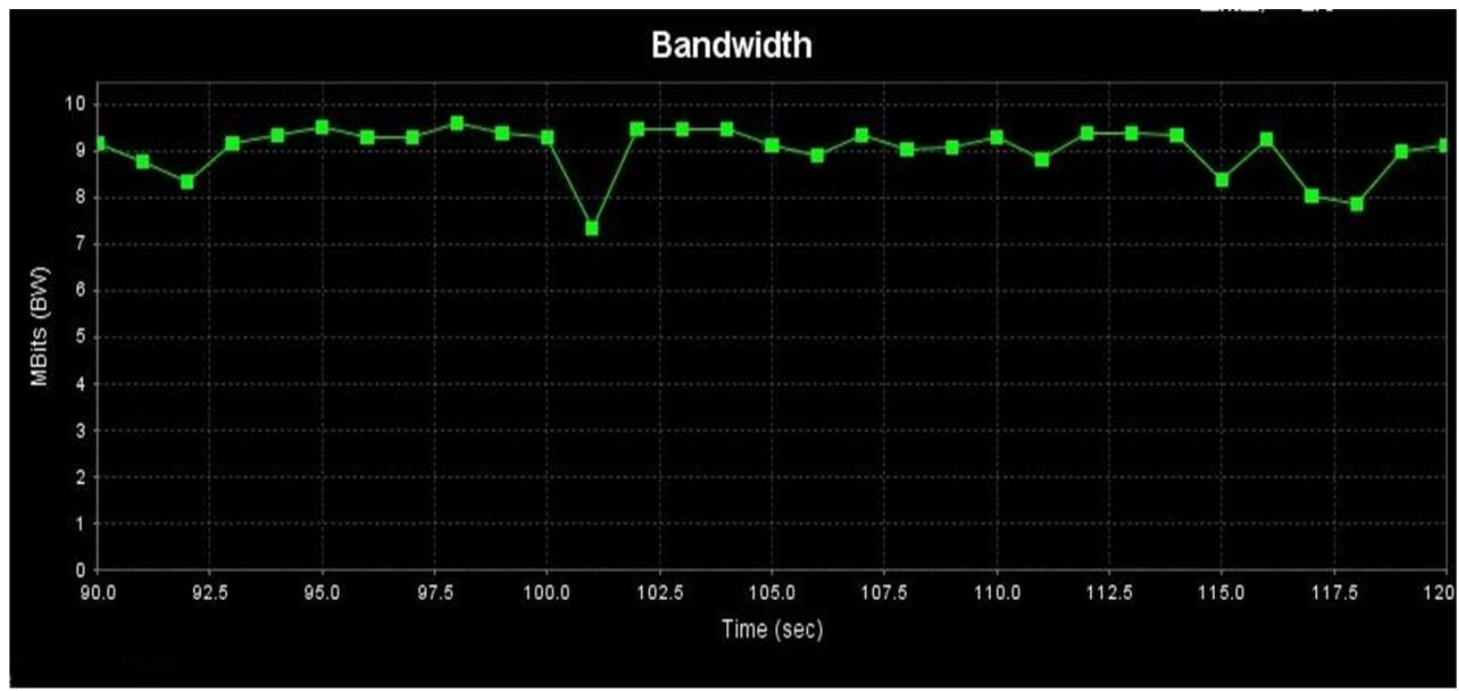

(a)

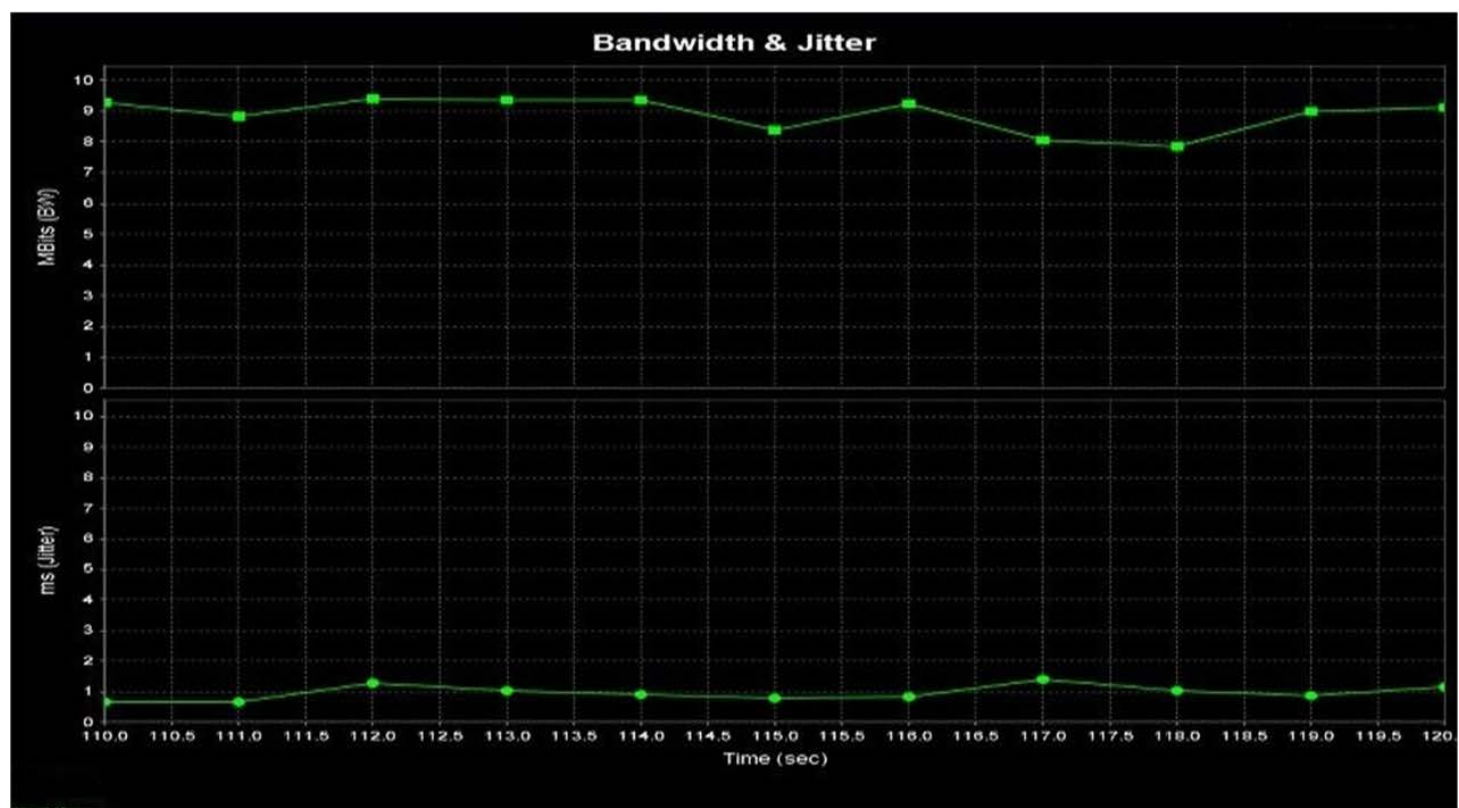

(b)

Fig. 16 Link test for Bandwidth UDP in 1 thread:

(a) Client interface,

(b) Server interface. 
ICSIST/NTUH/YZU Databank / Databank

Home

Introduction

Databank

COP DAT

OPERATION ROOM DATA

Publications

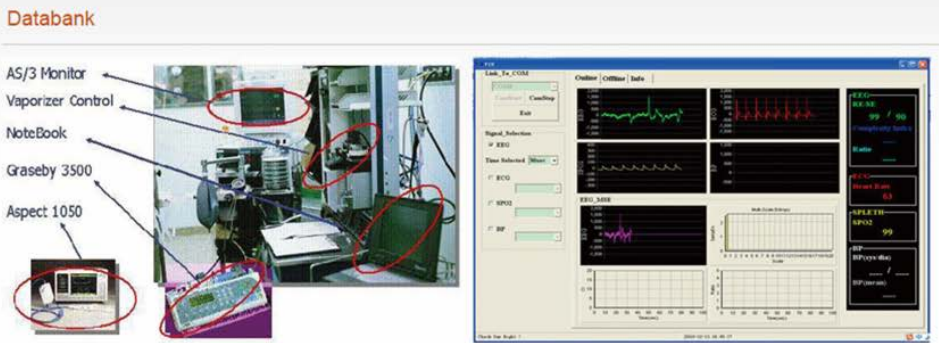

Biomedical Engineering Group

The Biomedical Engineering Research Group is working in the areas of anaesthesia, intensive care unit, brain wave nalysis, patient simulator and human performance.

Monitoring and Control of Anaesthesia:

The aim of this project is to develop an intelligent integrated control system that can monitor and assess the balanced anaesthesia, and control the drug via intravenous or inhalational infusions to maintain a constant level of muscle relaxation, unsciousness (i.e., depth of anaesthesia) and pain relif.

Intensive Care Unit:

The aim of this project is to develop computer based algorithms for monitoring and controlling patients in the neurosurgical intensive care unit

Brain Wave Analysis:

The aim of this project is to use quantitative parameters and qualitative analysis to analyse the stage from awake to early eep in order to develop a monitoring system using EEG signis for automobile operations to protect public health and safety. Bioinstrumentation Design:

The aim of this project is to use biomedical sensors, analog circuits, filtering techniques, and small-scale embedded systems to design different medical instrumentation, such as EKG, EMG, and EEG.

Patient Simulator:

The aim of this project is to develop a patient simulator automatically responded to the medical doctor's operation to educate the junior doctors to handle the incidents correctly not to endanger the real patients before clinical trials.

Fig. 17 Databank server. 